\title{
The effects of activity related fatigue on dynamic postural control as measured by the Star Excursion Balance Test
}

Timothy A. Kulpa

West Virginia University

Follow this and additional works at: https://researchrepository.wvu.edu/etd

\section{Recommended Citation}

Kulpa, Timothy A., "The effects of activity related fatigue on dynamic postural control as measured by the Star Excursion Balance Test" (2006). Graduate Theses, Dissertations, and Problem Reports. 2454.

https://researchrepository.wvu.edu/etd/2454

This Thesis is protected by copyright and/or related rights. It has been brought to you by the The Research Repository @ WVU with permission from the rights-holder(s). You are free to use this Thesis in any way that is permitted by the copyright and related rights legislation that applies to your use. For other uses you must obtain permission from the rights-holder(s) directly, unless additional rights are indicated by a Creative Commons license in the record and/ or on the work itself. This Thesis has been accepted for inclusion in WVU Graduate Theses, Dissertations, and Problem Reports collection by an authorized administrator of The Research Repository @ WVU. For more information, please contact researchrepository@mail.wvu.edu. 
The Effects of Activity Related Fatigue on Dynamic Postural Control as Measured by the Star Excursion Balance Test

\section{Timothy A. Kulpa, BS, ATC}

Thesis submitted to the School of Physical Education at West Virginia University in partial fulfillment of the requirements for the degree of

\section{Master of Science}

In

Physical Education

(Athletic Training)

\section{Michelle A. Sandrey, Ph.D., ATC, Chair \\ Sam Zizzi, Ed.D. \\ Matthew Lively, DO}

School of Physical Education

Morgantown, West Virginia

2006

Keywords: fatigue, dynamic postural stability, athletes 


\begin{abstract}
The Effects of Activity Related Fatigue on Dynamic Postural Control as Measured by the Star Excursion Balance Test

Timothy A. Kulpa, BS, ATC

The purpose of this study was to examine how dynamic postural control as measured by the Star Excursion Balance Test, was affected by an activity related fatiguing protocol. Forty male and female students from West Virginia University volunteered to participate. They completed the pre-test in all eight directions with the average of the three trials calculated for each direction. They then performed a functional fatiguing protocol that lasted 20 minutes with their rating of perceived exertion measured before, during and after. After completing the fatiguing protocol the post-test on the SEBT was in all eight directions. There was a significant interaction between time and direction and significant main effects for time and direction were also observed. A significant main effect for RPE was observed. The results of this study indicate that a fatiguing protocol will contribute to a significant decline in dynamic postural control when measured by the SEBT.
\end{abstract}




\section{ACKNOWLEDGEMENTS}

First, I would like to thank my parents for teaching me never to give up even when things are difficult. They have taught me the right way to live my life and their love and support has always been the driving source behind my hard work, and determination. Without their guidance I would not be in the position I am in today. Mom and Dad, thank you for all that you have done and continue to do for me.

I would like to thank my fiancé Stephanie Johnson, without her love and support I don't know how I would have gotten through graduate school. You were always there to listen even when you didn't understand what I was talking about. The distance apart has not been easy the last two years, but we made it work and I thank you for your patience and understanding.

I would like to thank Dr. Sandrey for granting me the opportunity to attend graduate school at West Virginia University. You helped me in all aspects of this project from brainstorming through the last day when it was finally complete. Your advice and ideas as well as the time and effort you sacrificed helped make this project what it is today.

I would like to thank Dr. Lively. There is no better team physician to work with. You taught me so much in my two years here. I thank you for taking time out of your very busy schedule to be a part of my committee; I really appreciate your effort and guidance.

I would like to thank Dr. Sam Zizzi for his time and effort in agreeing to be on my committee. You are one of the best professors I have ever had and were able to make statistics fun. I hope one day that I am able to have as good of a relationship with SPSS as you do.

I would like to thank all of the undergraduate and first year graduate students that volunteered for this study. Giving up an hour of your time is not easy, especially when it involves working yourself to exhaustion. I appreciate all of your effort and without you this project would have never been completed.

Finally to the entire West Virginia University Athletic Training Family, I thank you for the opportunity to work at this prestigious university during possibly two of the best years of WVU athletics. All of your guidance and support has transformed me into a professional. I will never forget then things I learned here, whether it be professional or personal, these experiences have been one of a kind. I have formed some great relationships here and I will truly miss everyone here as I move on with my professional career. WVU Athletic Training is a family and I am truly proud to say I am a Mountaineer! 


\section{TABLE OF CONTENTS}

ACKNOWLEDGEMENTS ..................................................

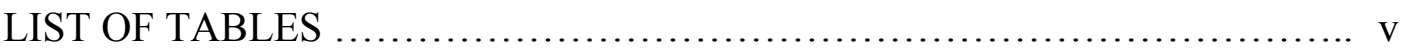

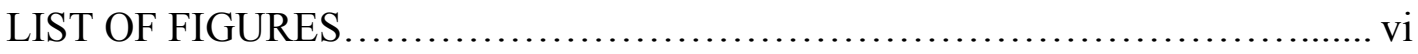

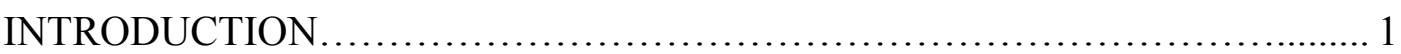

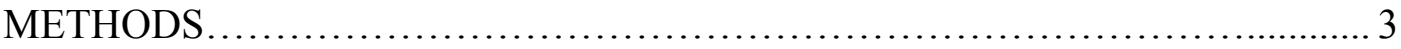

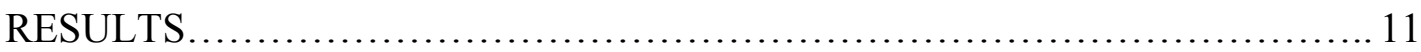

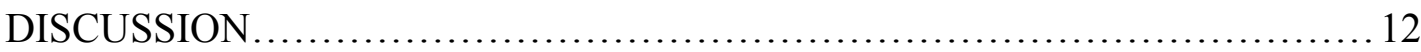

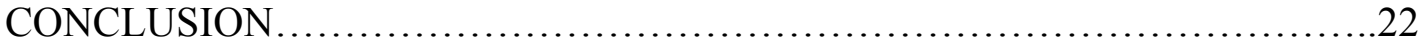

REFERENCES............................................................. 23

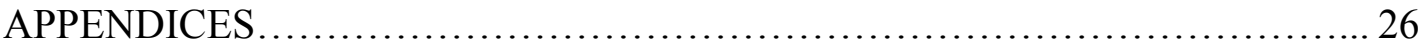

APPENDIX A: THE PROBLEM..........................................2 27

APPENDIX B: LITERATURE REVIEW ................................35

APPENDIX C: ADDITIONAL METHODS..............................69

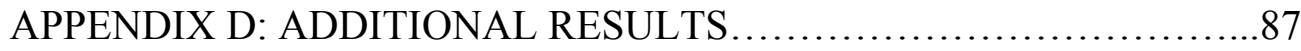

APPENDIX E: RECOMMENDATIONS FOR FUTURE RESEARCH..... 89

ADDITIONAL REFERENCES........................................... 90 


\section{LIST OF TABLES}

Table

B1. Isokinetic Fatiguing Protocols..................................... 58

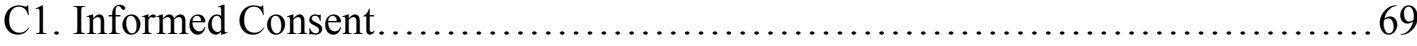

C2. Protected Health Information Release.................................... 74

C3. Demographic/Injury History Questionnaire.............................. 76

C4. Dynamic Postural Control Test using the Star Excursion Balance Test......... 78

C5. Pre-Test Data Collection Sheet.......................................... 79

C6. Fatiguing Protocol..................................................... 80

C7. Post-Test Data Collection Sheet......................................... 81

C8. Rating of Perceived Exertion (15 point Borg scale) $\ldots \ldots \ldots \ldots \ldots \ldots \ldots \ldots \ldots \ldots . \ldots 2$

C9. Rating of Perceived Exertion Data Collection Sheet........................ 82

D1. Descriptive Statistics for Star Excursion Balance Test..................... 87

D2. Within subjects ANOVA for the Star Excursion Balance Test............... 87

D3. Pairwise Comparisons for the Star Excursion Balance Test.................. 87

D4. Descriptive Statistics for Rating of Perceived Exertion.................... 88

D5. Within subjects ANOVA for Rating of Perceived Exertion................... 88

D6. Pairwise Comparisons for the Rating of Perceived Exertion................ 88 


\section{LIST OF FIGURES}

\section{Figure}

B1. Star Excursion Balance Test............................................... 66

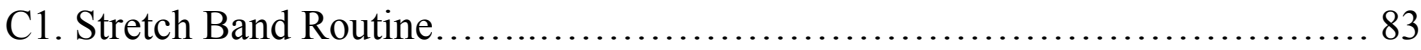

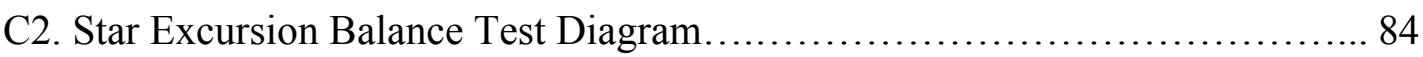

C3. Star Excursion Balance Test Pictures...................................... 85

C4. Fatiguing Protocol Diagram................................................ 86 


\section{INTRODUCTION}

In the last five years, a well publicized research area in the literature is the relationship between postural control and fatigue. Specifically, postural control can be defined as either static (maintaining a position with minimal movement), semi-dynamic (maintaining a position while the base of support moves), or dynamic (maintaining a stable base of support while completing a prescribed movement) ${ }^{1-6}$ Of the three, static and semidynamic measurements have been used the most in past and present studies. In addition a fatiguing protocol is used to simulate an injury model. For example, Gribble and $\mathrm{Hertel}^{7}$ looked at the effects of local muscle fatigue on static postural control when measured by a thirty second unilateral stance test. Although a good base of knowledge for researchers in the development of an injury model, most athletes do not participate in a static environment. Since most sporting events are dynamic in nature, they require a unique and complex coordinated effort from various systems of the body to allow the athlete to participate at his/her peak level.

With this in mind a recent publication by Gribble et al. ${ }^{8}$ looked at the effects of dynamic postural control and how it responds to a fatigued state. Dynamic postural control is defined as the "completion of a functional task without compromising one's base of support." 2 This form of postural control is important in almost any sporting activity whether it be shooting a foul shot in basketball, hitting a backhand in tennis, or kicking a soccer ball. Having proper control of your body allows the individual to concentrate totally on the functional task at hand. In addition to assessing balance, studying dynamic postural control also adds additional demands of proprioception, range of motion, and strength while attempting to maintain an upright and steady posture. ${ }^{2}$ The only test currently in the 
literature that truly stresses the dynamic balance of healthy individuals is the Star Excursion Balance Test (SEBT). ${ }^{2,3,9-11}$ This test is said to provide a significant challenge to the athlete's postural control system and shows strong intra-tester reliability, and is sensitive for the screening of musculoskeletal injuries. ${ }^{2,9-11}$

One problem with the current research is that it often uses a repeated isokinetic exercise, or an isometric hold to fatigue the muscle or muscles being examined. Although an easy standardized way to fatigue a muscle or muscle group, these activities are usually not functional or sport related in anyway. However, there are few times in any sport where a player will be standing in a static isometric plantarflexed position until they can not hold it anymore, and then play in a game. Since researchers have been able to develop this vast base of support knowledge in this area, it is time to take the next step. Research needs to support a more functional or sport-related fatiguing protocol, to observe how the rigors of athletic competition affects the dynamic postural control system of the body. There is a need to determine if the coordinated movements involved in sports along with cardiovascular endurance combine to produce a different level of fatigue that can have a detrimental effect on dynamic postural control. It appears that with the lack of current research linking dynamic postural control and fatigue, and the current concepts in rehabilitation that one would want to examine the possible connection between overall body fatigue and how it interacts with dynamic postural control. Therefore the purpose of this study is to examine how dynamic postural control as measured by the Star Excursion Balance Test, is affected by an activity related fatiguing protocol. 


\section{METHODS}

This study consisted of two factorial designs. The first was a 2 × 8 factorial design for the Star Excursion Balance Test. The independent variables are time (pre and post fatigue) and direction (Anterior, Anteriolateral, Anterolateral, Lateral, Posterolateral, Posterior, Posteromedial, Medial, Anteromedial). The dependent variables are the scores on each of the eight reach directions of the Star Excursion Balance Test. To measure fatigue, a 1 x 3 factorial design was used for Rating of Perceived Exertion (RPE). The independent variable is time (pre, middle, and post fatiguing protocol), and the dependent variable is the RPE value.

Subjects

Forty healthy and physically active male $(n=18$, age $=20.6 \pm 1.4$ years, height $=$ $181.6 \pm 7.8 \mathrm{~cm}$, and mass $=76.4 \pm 14.6 \mathrm{~kg})$ and female $(\mathrm{n}=22$, age $=21.1 \pm 1.8$ years, height $=169.7 \pm 8.7 \mathrm{~cm}$, and mass $=60.8 \pm 10.8 \mathrm{~kg}$ ) students from the undergraduate and graduate athletic training programs at West Virginia University volunteered to participate. Subjects were excluded if they had a history of multiple ankle sprains (three or more significant sprains within the last year), an ankle sprain or injury within the last six months, a history of multiple knee injuries (three or more within the last year), a knee injury or surgery within the last year, a history of multiple hip injuries (three or more within the last year), a hip injury or surgery within the last six months, history of multiple concussions (three or more in their life time), a concussion within the last six months, any neurological or central nervous system deficits, or was currently taking any medication that could affect the nervous system. Subjects read and signed a written consent approved by the university 
before beginning the study. The study was approved by the West Virginia University

Office of Research Compliance for the Protection of Human Subjects.

Instrumentation

Being able to measure postural control is important in establishing levels of function for injury prevention and rehabilitation. ${ }^{2}$ Testing dynamic postural control requires completion of a functional task while maintaining a base of support. Assessing dynamic postural control has its advantages because it takes into account the additional demands of ROM, proprioception, and strength while trying to maintain the base of support. ${ }^{2,3,9,11}$ Initially introduced by Gray, the SEBT is a relatively simple and versatile test.

To begin the test, the athlete's leg length should be measured using the true leg length method of measurement (Anterior Superior Iliac Spine (ASIS) to medial malleolus) to normalize the reach based on the length of the subject's legs. Normalizing the data to leg length allows the clinician to compare data between participants and genders without worrying about the height of the subjects. ${ }^{2,9,11}$ Next, six practice trials are performed to learn the task and to develop balance strategies., ${ }^{2,39-11}$ These six practice trials were noted by Kinzey ${ }^{9}$ to decrease the learning effect for this relatively difficult task. For research purposes each direction is best performed three times, taking the average of the three trials for each direction. Also, the test is performed in the clockwise (if left leg is dominant) or counter-clockwise (if right leg is dominant) direction with a randomized starting point that can be pre-determined by the clinician. ${ }^{2,9,12}$ The dominant leg can be identified as the leg the subject is most likely to use to kick a soccer ball. 
As with any type of movement pattern or balance event, different postural alignments can affect the subjects score on the SEBT. One major problem might be the biomechanics and physical make-up of the stance foot. In 2005 , Cote et al. ${ }^{13}$ examined the effects of the pronated and supinated foot on static and dynamic postural stability. They suggested that an excessively supinated foot characterized by a high arch and hypomobile midfoot, may not adapt to the underlying surface, increasing the demand on the surrounding musculoskeletal structures to maintain postural stability. On the other hand, they stated that an excessively pronated foot, which is characterized by a hypermobile midfoot, and a flattening of the medial arch, may place a high demand on the neuromuscular system to stabilize the foot and maintain an upright posture. When they examined dynamic balance and foot type with the SEBT, they found that supinators reached further in the lateral and posterolateral directions. A supinated foot would place greater amounts of pressure on the lateral aspect of the foot, producing a reach deficit in the medial direction. On the other hand, pronators reached further in the anterior direction than both supinators and neutrals, and further than neutrals in the anteromedial direction. This again can be explained by the nature of the deformity. Pronators tend to collapse down on the medial aspect of the foot and therefore have a reduced ability to maintain a rigid support system during full weight bearing. Because of the medial foot deviation and the greater midfoot mobility, it seems logical that there would be differences in reach.

Hip strength and range of motion, ankle range of motion especially in dorsiflexion, and strength differences especially in ankle dorsiflexion may affect the measurements. Although Gribble and $\mathrm{Hertel}^{3}$ did not find any significant effects from ankle dorsiflexors strength or ankle and hip range of motion, one can still hypothesize that in a larger 
population these factors may affect reach distance. For example, an athlete who suffers from limited dorsiflexion due to tight gastrocnemius and soleus muscles would not be able to dorsiflex enough, hence decreasing reach distances. Likewise someone who suffers from limited hip flexion may also not reach as far as someone who has good flexibility at the hip. In addition to available range of motion, the subject must also be able to control the range of motion through muscular contractions. Having a strong anterior tibialis muscle may allow the subject to control their movements in a greater amount of dorsiflexion, thus allowing them to reach further.

There are a plethora of tests developed to assess dynamic postural control in the pediatric and geriatric populations, while the SEBT appears to still be the only test used on an otherwise healthy population. ${ }^{2,3}$ The SEBT has been shown to be reliable. ${ }^{9,12}$ The intrarater reliability of the SEBT has been shown to be moderate by Kinzey and Armstrong $^{9}\left(\mathrm{ICC}_{2,1}=.67\right.$ to .87$)$ using the anteriolateral, posteriolateral, anteromedial, and posteriomedial directions only, and Hertel et $\mathrm{al}^{12}\left(\mathrm{ICC}_{2,1}=.81\right.$ to .96 . Although the SEBT is reliable, a true measure of validity has yet to be performed for this instrument. This is due to the fact that it cannot be compared to a gold standard. In order to perform a validity study, data would have to be compared to a force plate which is the closest thing to a gold standard that we currently have. In my study the reliability values were $\left(\mathrm{ICC}_{2,1}=.896 ; .838\right.$ to .938$)$ for pre-test and $\left(\mathrm{ICC}_{2,1}=.970 ; .954\right.$ to .982$)$ for post-test.

Kinzey and Armstrong ${ }^{9}$ suggested that to bring the intraclass correlation (ICC) above .86 that each participant should partake in six practice sessions. They commented further saying that the reliability results that they obtained were moderate at best and that a learning period is necessary prior to being evaluated on the SEBT. ${ }^{9}$ Hertel et al. ${ }^{12}$ also 
agreed with this, and further suggested that "participants need to perform six practice trials in each of the eight directions before any data is recovered." These practice sessions that both Kinzey and Armstrong ${ }^{9}$ and Hertel et al. ${ }^{12}$ suggested allow the participant to familiarize themselves with the test and decide on their own unique reaching strategy.

In this study I used the Rating of Perceived Exertion (RPE) values to determine how much fatigue the subjects were feeling. Some question if this is an adequate measure and if it can quantify the level of fatigue a participant is experiencing. In an attempt to quantify the amount of fatigue using RPE, both Susco et al. ${ }^{14}$ and Wilkins et al. ${ }^{15}$ used the 15 point Borg scale as is the case with many research groups that study RPE. The theories behind how RPE determines the level of fatigue lies deep in exercise physiology. RPE and $\mathrm{VO}_{2}$ max are very highly correlated, and because of this the RPE scale may be used as a substitute to determine exercise intensity. ${ }^{16}$ Seliga et al. ${ }^{16}$ was able to document that perceived exertion scores increased significantly with an increase in workload, with a heavy workload represented by an RPE value of 14-16. In addition, some researchers have concentrated on correlating RPE with $\mathrm{VO}_{2} \max$. Robertson et al. ${ }^{17}$ found that in a group of physically active males, RPE was measured during cycling, treadmill exercise, and simulated skiing at various levels of $\mathrm{VO}_{2} \max$. The RPE values increased significantly with the increase in $\mathrm{VO}_{2}$ max percentage.

Procedures

To begin there was a general informational meeting in the Athletic Training Research Laboratory in the Coliseum. During that meeting a demonstration and general overview of the research was presented. An informed consent form (Table C1), Protected Health Information Release (Table C2), and demographic and injury history form (Table 
C3) were handed out. The subjects were asked to read and sign the informed consent and complete the demographic and injury history questionnaire if they were interested in participating in the study. After the meeting, the principle investigator reviewed the forms and determined who met the inclusion criteria and not any of the exclusion criteria. A second meeting was held to inform the participants who was selected and to tell them when they needed to report for testing, proper dress code for the testing (clean athletic shoes, shorts, t-shirt), and what was to be expected of them during the testing session.

Subjects were tested using the Star Excursion Balance Test (SEBT), which tests dynamic postural control prior to completing an activity related fatiguing protocol, and directly after completing the activity related fatiguing protocol. The activity related fatiguing protocol consisted of seven stations of functional activities designed to fatigue the participant to a Rating of Perceived Exertion (RPE) level of 15 or higher on the 15point Borg scale. ${ }^{14,15}$

Star excursion balance test: The participants reported to the Coliseum main court at the time and date given to them at the second orientation meeting. Once they arrived the participants had the length of their dominant leg measured in centimeters to normalize the data to leg length. Next the subjects had five minutes to warm-up on a stationary bicycle at level 2. After the warm-up period, the subjects performed a stretch-band routine for five more minutes isolating the hamstrings, IT Band, Groin, Gluteals, Quadriceps, hip flexors and gastroc/soleus. (Figure C1). Next they performed the pre-test (non-fatigued) Star Excursion Balance Test. The test was first explained, and then demonstrated by the principal investigator. The SEBT involves taping a star pattern with eight projections (excursions) each at a $45^{\circ}$ from each other, on an even surface (Figure C2, Figure C3). 
Subjects placed their dominant foot on the middle of the star, while using their nondominant foot to reach as far as possible in each of the eight directions without compromising their base of support (Table C4). The subject's foot dominance was identified by asking them which foot they would use to kick a soccer ball. They then touched their foot down on the farthest point they could reach as lightly as possible using the most distal portion of their foot. This mark was recorded by the principle investigator and measured in centimeters as the subject returned to the middle and to bilateral stance while maintaining their balance. The subject was allowed six practice sessions in each of the eight excursions with 15 seconds rest in between excursions followed by a five minute rest period. After the rest period, the principle investigator chose a starting excursion at random by drawing out of a hat and the subject moved in a clockwise direction (if left leg is dominant) or counter-clockwise (if right leg is dominant) until they completed all eight excursions. For each excursion the subject performed three trials with the average of the three recorded as their pre-test score for that particular excursion (Table C5). Trials were discarded and repeated if the reach foot was used to provide considerable support during the motion, the stance foot was lifted from the center of the grid, or if the subject was unable to maintain their balance throughout the excursion. ${ }^{9}$

Fatigue protocol: The fatiguing protocol (Figure C4, Table C6) consisted of seven stations each designed to fatigue the subject by using different energy systems to represent a sport-related fatigue. Each station was timed by a Timex Ironman Triathlon watch. Just prior to the first station, the principal investigator took the RPE reading from the participant (Table C8, Table C9). 
Station one consisted of a five-minute moderate jog at the subjects self-selected pace. This jog took place around the perimeter of the gym in order to help the participant maintain a steady pace. Station two consisted of three minutes of sprints up and down the length of a basketball court. Station three was two minutes of push-ups. After station three was completed, the RPE measurement was taken as station three is considered the half-way point. Station four consisted of two minutes of sit-ups. Station five was three minutes of 12 inch step-ups. Station six consisted of another three minutes of sprints up and down the basketball court, and station seven was a two minute run at as fast a pace as the participant could maintain for the entire two minutes. ${ }^{14,15}$ A third RPE (Table C8, Table C9) was recorded as soon as the subject completed the last station. Adequate fatigue has been deemed to be an RPE of 15 which represents $75 \%$ to $90 \%$ maximum oxygen consumption..$^{14,15}$

Immediately after taking the RPE, the subject completed the post-test (post-fatigue) Star Excursion Balance Test. The procedure was similar as the pre-test with the only exception being the starting excursion was again chosen at random by the principal investigator. Once the subject had completed the final trial the subject was free to leave. Data Analysis

The data (three trials in each excursion, the average of the three trials, for pre and post tests, and their pre, middle, and post RPE values) was recorded on coded sheets. The scores obtained from the pre and post SEBT were then normalized to leg length by dividing the distance reached by the measure length of the leg (excursion length/leg length $\mathrm{x} 100$ for a percentage of an excursion distance in relation the subject's leg length). ${ }^{3}$ Upon test completion, all data and scores were entered into a spreadsheet on SPSS. 
Statistical Analysis

Descriptive statistics consist of means and standard deviations for all subjects in each of the eight directions for pre and post test, as well as for the pre, middle, and post RPE scores. A two-way repeated measures ANOVA was used to determine the main effects and interaction between time and direction for the excursions of the Star Excursion Balance Test. Post-hoc testing consisted of eight pairwise comparisons, one for each reach direction for pre and post testing. A one-way repeated measures ANOVA was used to determine if a difference existed for Rating of Perceived Exertion. Post-hoc testing consisted of three pairwise comparisons (pre-test to mid-test, pre-test to post-test, and midtest to post-test) to determine which changes were significant. The $p$ value for all tests was set at $\mathrm{p} \leq 0.05$. All data was analyzed using SPSS Version 14.0 for Windows. (SPSS Inc.

Chicago, IL).

\section{RESULTS}

Star Excursion Balance Test

All descriptive statistics for each excursion are presented in Table D1. Results from the $2 \times 8$ repeated measures ANOVA revealed a significant interaction between time and direction $\left(\mathrm{F}_{1,39}=9.487, \mathrm{P}=.004, \mathrm{ES}=.196, \beta=.862\right)$. Significant main effects for time $\left(\mathrm{F}_{1,39}=554.675, \mathrm{P}<.001, \mathrm{ES}=.934, \beta=1.000\right)$ and direction $\left(\mathrm{F}_{1,39}=49.010, \mathrm{P}<.001, \mathrm{ES}\right.$ $=.557, \beta=1.000)$ were also observed. Full results can be observed in Table D2. A pairwise comparison was completed and found to be significant for all eight reach directions from pre-test to post-test for Anterior $(\mathrm{P}<.001)$, Anterolateral $(\mathrm{P}<.001)$, Lateral $(\mathrm{P}<.001)$, Posterolateral $(\mathrm{P}<.001)$, Posterior $(\mathrm{P}<.001)$, Posteromedial $(\mathrm{P}<.001)$, Medial, $(\mathrm{P}<.001)$, Anteromedial $(\mathrm{P}<.001)$. Results are found in Table D3. 


\section{Rating of Perceived Exertion}

Descriptive statistics for each of the three rating of perceived exertion (RPE) measurements can be observed in Table D4. Results from the 1 x 3 repeated measures ANOVA revealed a significant main effect for $\mathrm{RPE}\left(\mathrm{F}_{1,39}=2436.147, \mathrm{P}<.001, \mathrm{ES}=.984\right.$, $\beta=1.000$ ) and can be observed in Table D5. A pairwise comparison showed that pre-test to mid-test $(\mathrm{P}<.001)$, pre-test to post-test $(\mathrm{P}<.001)$, and mid-test to post-test $(\mathrm{P}<.001)$ were all statistically significant. (Table D6.)

\section{DISCUSSION}

The main objective of this study was to examine if a 20 minute activity related fatiguing protocol would affect an individual's dynamic postural control when measured by the Star Excursion Balance Test (SEBT). The results of this study indicated that there is indeed a significant interaction between reach direction and time which indicates that the post-test SEBT reach distances were less than the pre-test measures in all eight excursion directions. In addition there was also a significant difference in time when it came to reporting the level of exertion on the rating of perceived exertions scale. As the SEBT went on, the individuals reported feeling more fatigued, with higher levels recorded between the mid-test measure and the post-test measure. The findings in this study support all eight of the experimental hypotheses stating that the pre-test reach distances would be significantly greater than the post-test reach distances in all eight of the excursions (anterior, anterolateral, lateral, posterolateral, posterior, posteromedial, medial, anteromedial). Fatigue Protocol

To begin the discussion, it must be known that this study is the first to report on an activity related fatiguing protocol and dynamic postural control. A majority of other 
fatiguing studies have evaluated static postural control ${ }^{7,10,14,15,18}$ or joint position sense at the ankle ${ }^{19,20,21,22}$ with or without a fatiguing protocol. These studies that used a local fatiguing protocol followed either an isokinetic fatiguing protocol, ${ }^{8,23,24}$ a repeated lifting protocol, ${ }^{25}$ or an isometric fatiguing protocol. ${ }^{19,20,22,26,27,28}$ Few studies, if any exist in the literature that use a central or general fatigue protocol to evaluate dynamic postural control. Lepers et al. ${ }^{29}$ used a cycling bout protocol, but did not examine dynamic postural control. Susco et al. ${ }^{14}$ and Wilkins et al. ${ }^{15}$ used this functional fatiguing protocol, but evaluated the subjects performance on the Balance Error Scoring System (BESS), a measure of static balance.

Although recently there has been extensive research on fatigue and dynamic postural control as measured by the Star Excursion Balance Test (SEBT), there is limited research available examining a sport-related fatiguing process and the effects it would have on dynamic postural control. An activity related fatiguing process would involve an exercise bout that would more closely resemble a "typical" practice or game and would fatigue both local and central processes instead of just concentrating on a single muscle or muscle group. ${ }^{14,15}$ This fatiguing process would include various types of exercise in an attempt to fatigue the athlete including but not limited to isokinetic exercise, isotonic exercise, plyometric and agility exercises, as well as testing their cardiovascular endurance. $^{15}$

One of the factors in developing and designing a central or general fatiguing protocol is that it actually is taxing to all energy systems. Although fatigue substrates and metabolites were not determined in this study or the Wilkins et al. ${ }^{15}$ or Susco et al. ${ }^{14}$ studies, Susco et al. ${ }^{14}$ determined that an activity of this nature does cause fatigue and that 
fatigue lasts at least twenty minutes. The post-test SEBT took on average 10 minutes to complete, with the longest time being 13 minutes. If one considers the 20 minute fatigue time span in the Susco et al. ${ }^{14}$ study, the participants should still have been fatigued and were still reporting feelings of fatigue. To ensure fatigue occurred in all three studies, the rating of perceived exertion was used to determine the participant's level of fatigue. According to both studies, adequate fatigue was deemed to be an RPE of 15 or higher. In my study, all 40 participants reported an RPE above $15(17.33 \pm .764)$ on the post-test RPE collection.

In an earlier study Mahon et al. ${ }^{30}$ examined RPE during a 10 minute graded cycle ergometer test. The RPE values that they reported were $10.2 \pm 1.2$ at the 5 minute mark ( $80 \%$ ventilatory threshold) and $15.8 \pm 1.7$ at the 10 minute mark $(120 \%$ ventilatory threshold). These RPE values are very similar to the RPE values for mid-test $(12.55 \pm 1.1)$ and post-test $(17.33 \pm 0.8)$ reported in my study. This activity related fatiguing protocol was chosen in an attempt to replicate the fatigue an athlete would experience during the course of a practice or competition. I feel confident that the changes in the RPE scores (all found to be significant, $\mathrm{P}=.000$ ) demonstrates that my subjects were fatigued to a level that represented between $75 \%$ and $95 \%$ of maximal oxygen consumption $\left(\mathrm{VO}_{2} \max \right)$ and that the fatigue is what caused the significant decrease in reach distances between the pre-test and post-test SEBT.

Susco et al. ${ }^{14}$ stated that using the twenty-minute fatiguing protocol resulted in a significant decrease in Balance Error Scoring System (BESS) performance in all test groups, with the exertion group having the greatest effect on the tandem and single-leg stance conditions. In addition they found that all subjects had recovered by post-test 2 , 
which was administered 20 minutes after the cessation of the exertion protocol. Wilkins et al. ${ }^{15}$ used the same protocol and stated that there was a significant increase in total errors from pretest to posttest in the fatigue group $(14.36 \pm 7.73$ versus $16.39 \pm 4.32)$. They also noted that the RPE scores were significantly different between the fatigue and control groups at the middle $(13.29 \pm 1.59$ versus $6.23 \pm 0.83)$ and end $(15.66 \pm 2.38$ versus $6.15 \pm$ $0.55)$ of the fatigue or rest period.

Although this study reported on dynamic postural control, reach distance decreases were observed after fatigue. It has been observed that during static postural control measures, the input from the plantar cutaneous receptors is used to aid in afferent input, thus assisting in maintaining a stable base of support. ${ }^{8}$ Since we know that using orthotics helps improve static postural control, the use of these plantar cutaneous receptors is important. ${ }^{6}$ Research from van Deursen et al. ${ }^{31}$ has demonstrated a link between the plantar cutaneous receptors and the biarticulate muscles of the thigh, creating an important link to the maintenance of an upright and stable posture. This relationship to muscle activation of the muscles controlling the hip and knee is significantly decreased in individuals suffering from cutaneous deficits to the plantar surface of the foot, and thus these patients demonstrated reduced static postural control. It is possible that these neuromuscular deficits could follow the constant changing of foot posture when attempting to reach during the SEBT. It was noted that during the post-test trials, the subject were constantly rocking different portions of their foot to maintain balance when getting towards the end range of the reach distance. This constant posture shifting could have caused a decrease in the cutaneous plantar receptors ability to link to the proximal muscles and thus affected the neuromuscular control of the entire lower kinetic chain. ${ }^{31}$ 
As demonstrated by most of the studies described, fatigue does in fact have an impact on postural control. How large of an impact it has is a question that is always under investigation. Fatigue can have an impact on static postural control, semi-dynamic postural control and well as dynamic postural control. The effects of local and peripheral on local muscles such as the ankle plantarflexors when standing on the tip toes $23,24,26,27$ is not exactly clear how local muscular fatigue affects postural control. Fatigue is the reduction in

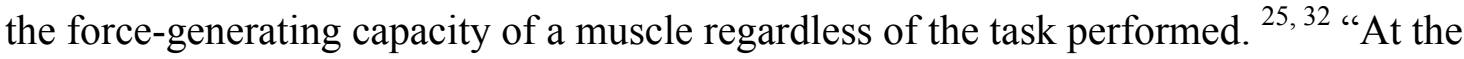
peripheral level the pre- and postsynaptic mechanism and action potential sites are affected including a failure in the transmission of the neural signal or a failure of the muscle to respond to neural excitation." 25 At the central level, fatigue may also cause a failure of the excitation of motoneurons due to changes in the nervous system. ${ }^{25}$

The contribution of the joint and mechanoreceptors also is controversial. It appears that the greatest contribution to proprioception is from the muscle receptors, specifically the muscle spindles, and golgi tendon organs. Since fatigue protocols affect muscle tissue more than articular receptors, diminished proprioception, and therefore postural control may conceptually be thought of as secondary to loss of muscle receptor input. ${ }^{33}$ It is also theorized that muscle fatigue may impair the proprioceptive and kinesthetic properties of joints by, "increasing the threshold of muscle spindle discharge, disrupting afferent feedback, and subsequently altering conscious joint awareness."7

The information presented on functional fatigue indicated that general fatigue also plays a role in altering postural control. Although the effects of functional are much more difficult to quantify, the authors represented have been able to conclude that long bouts of 
cycling induce neuromuscular fatigue and that 30 minutes of station exercise can alter balance performances on the Balance Error Scoring System (BESS). ${ }^{14,15,29}$

In addition, fatigue that is peripheral in nature can be the result of activation of the nociceptors by the metabolic products of muscular contraction. These metabolites include bradykinin, arachidonic acid and prostaglandin $\mathrm{E}_{2}$, potassium, and lactic acid. ${ }^{22}$ These metabolites have been noted to have a direct impact on the discharge patterns of the muscle spindles, in addition to changing the afferent and efferent pathways via reflexes originating from the small-diameter muscle afferents (groups III and IV afferents). ${ }^{22,34}$ It is possible that the fatiguing protocol cause peripheral or local fatigue to occur around the muscles and joint receptors that allow movement for the SEBT. This fatigue then altered the body's ability to process the changing information to allow the participant to maintain a stable base of support.

In addition to local fatigue, central or general fatigue may also have an impact on dynamic postural control. The role that central fatigue plays to increase the perceived effort signal is easily understood. During an activity, the motor cortex is excited to recruit more motor neurons and muscles, thus driving them harder. ${ }^{35}$ If the participants perceived the exercise getting harder, perhaps they started to make unnecessary muscle movement as they recruited more muscle fibers and thus forcing them to work harder. In this case, it is important to look at a study by Li et at. ${ }^{36}$ which examined the H-reflex. Their study used an isokinetic fatiguing protocol and noted that muscle fatigue did indeed decrease motoneuron pool excitability. Further, attempting to perform a voluntary isometric contraction (like the ones necessary to perform the post-test SEBT) would need to incorporate the ability to 
recruit motoneurons and this would be decreased due to the fatiguing protocol's effect on the H-reflex. ${ }^{36}$

Another way to evaluate a fatigue protocol is to compare it to an injury model. When the body is in a fatigued state, it responds much the same as when it is injured. If this is the case, we can look at the research linking chronic ankle instability to postural control. It is theorized that when injured the joint receptors incur damage that delays the afferent conduction to recruit the corrective muscle contractions from the efferent signals in response to a perturbation. ${ }^{8}$ In our case, we do not have a specific injury that would damage the joint receptors, however it has been demonstrated that fatigue, especially peripheral fatigue can slow the propagation of the activation down the t-tubules, and therefore delay the activation of the contractile elements that generate force and power in response to the perturbation. ${ }^{37}$ In this case the perturbation is the body losing its balance or more specifically, the fatiguing protocol would alter the activation necessary to maintain a stable base of support while reaching out.

Fatigue and Dynamic Postural Control

The actual test of the Star Excursion Balance Test may be used to explain the differences. Many authors have identified specific excursions that are easier to perform than others. Specifically the posterior, posteromedial, and medial are usually identified as being the easiest to perform, where as the anterior, anterolateral, and lateral have traditionally been the most difficult to perform. ${ }^{2,3,8,9}$ In their study post hoc testing revealed that the lateral direction was by far the most difficult, and reach distances in the anterolateral direction were also significantly shorter than all of the other directions except lateral. They went further to say that the posterior and posteromedial directions seemed to 
yield the greatest reach distances, and were significantly longer than the other excursions. In my study there was a significant decrease in all eight reach directions. This was noted for the most difficult reach directions in the anterior, anterolateral, and lateral directions, and with the easier reaches in the posterior, and posteromedial directions.

Gribble et al. ${ }^{8}$ looked at the effects of fatigue and chronic ankle instability on dynamic postural control. They had all of the subjects complete five testing sessions in which saggital-plane kinematics and reaching distances were recorded while they performed the anterior, medial and posterior excursions of the Star Excursion Balance Test. They indicated that the involved side of the chronic ankle instability subjects had a significantly smaller reach distance and knee flexion angles for all three directions. They also noted that the fatiguing protocols decreases reach distance and knee flexion angles further. This is relevant to my study because the fatigue the occurred in my study could be compared to the chronic ankle instability group through the use of an injury model.

The SEBT is a valid and reliable tool in measuring dynamic postural control. In an attempt to normalize data, the test calls for normalizing the reach distances to leg length so the scores can be generalized to the population. However, one area that varies and is difficult to normalize is muscle strength and flexibility. The SEBT allows for cocontraction of the hamstrings and quadriceps throughout all directions of the test with the quadriceps most active during the three anterior excursions. The vastus lateralis is most active during the medial and posteromedial excursions most likely being the result of muscular stabilization that is performed against the varus force that is caused by the reach directions.

${ }^{11}$ With this in mind, performing the anterior and medially directed excursions uses eccentric control of the quadriceps. If a participant has poor eccentric control or strength of 
the quadriceps, this could lead to decreased reach distance on these directions of the SEBT.

${ }^{11}$ In addition the fatigue protocol could have had an affect on the con-contraction needed to correctly performed the SEBT.

In addition the biceps femoris is very active during the posterolateral, posterior, and lateral excursions. These directions cause an eccentric contraction of the hamstrings to resist the hip flexion moment caused by flexing the trunk to extend the leg backward. The fatiguing protocol that we used involved a lot of sprinting and running that would cause the hamstrings to contract eccentrically to slow the individual. If these muscles are eccentrically contracted repeatedly by the fatiguing protocol it would make these excursions especially difficult to perform. ${ }^{11}$

During the post-test SEBT, it was difficult for the individuals to maintain the single leg stance between the individual excursions. In addition, there were more errors noted during the post-test SEBT (21) versus the pre-test SEBT (2). During the fatiguing protocol, the participants were asked to perform many different movements that required both concentric and eccentric control of both the quadriceps and hamstrings. For example, the step-ups required eccentric control of the quadriceps thus contributing to the fatigued state of the quadriceps. It can be theorized that the eccentric contractions produced in the pretest SEBT and the eccentric contractions necessary to perform the SEBT, would combine to form a moderate to severely fatigued state.

Clinical Implications

With the trend of injuries possibly occurring during the later half of games and practices, perhaps more can be done to prepare these athletes for central and local fatigue. For example athletic trainers and strength and conditioning coaches could develop pre- 
season conditioning programs incorporating exercising in a fatigued state. If we can better train the athlete to operate in a fatigued environment, or limit the amount of fatigue an athlete is experiencing as a result of improved conditioning, then perhaps injuries may not be as correlated with the amount of fatigue that they are experiencing.

It is known that a change in afferent input from the peripheral receptors will cause a change in the neuromuscular control of the lower extremity. We also know that fatigue alters the afferent input from the muscle receptors. Combined, this leads to a decrease in the body's ability to control the lower limb. ${ }^{19}$ However, there has not been a direct link between fatigue-mediated changes in proprioception and changes in the body's ability to stabilize the lower limb. Skinner et al. ${ }^{38}$ noted that the capsular receptors may be responsible for the kinesthesia present in the absence of muscle-receptor function. For these reasons, capsular receptors and muscle receptors should be addressed through the use of weight-bearing proprioceptive exercises to increase the sensitivity and efficiency of the neuromuscular-control mechanisms before progressing to high-level strengthening exercises. $^{19}$

Even though recreationally active participants were used, there is some evidence that can be generalized to the athletic population. In fact the findings generated by this study could have potential implications for future clinical practice. In my study I focused on recreationally active participants in hopes that the results could be generalized to the competitive athletics population. Although it is not realistic to expect a coach to stop practice or decrease practice times because someone is experiencing fatigue which may lead to injury, this topic needs attention. To be realistic, most coaches could rearrange how practices are conducted. For example, a coach who is having three hour basketball 
practices could schedule the more difficult and higher risk activities in the first half of practice and leave the lower risk activities for the last hour when the athletes will be feeling the effects of fatigue.

\section{CONCLUSION}

The results of this study indicate that there is a significant interaction between distance reached on the Star Excursion Balance Test, and time (pre-test or post-test). When the participants were fatigued they demonstrated a decreased reach distance in all eight directions of the SEBT especially in the anterior, anterolateral, and lateral directions.

It is believed that the 20 minutes activity related fatiguing protocol combined effects of local (peripheral) fatigue and elements of central (general) fatigue to decrease the participant's dynamic postural control. How exactly this happened is not widely understood, and further research into the exact mechanism of fatigue on dynamic postural control is needed. 


\section{REFERENCES}

1. Guskiewicz KM, Perrin DH. Research and clinical applications of assessing balance. $J$ Sport Rehabil. 1996;5:45-63.

2. Gribble, P. The star excursion balance test as a measurement tool. Athl Ther Today. 2003;8(2):46-47.

3. Gribble P, Hertel J. Considerations for the normalizing measures of the star excursion balance test. Measurments Phys Educ Exer Sci. 2003;7:89-100.

4. Nakagawa L, Hoffman M. Performance in static, dynamic, and clinical tests of postural control in individuals with recurrent ankle sprains. J Sport Rehabil. 2004;13:255-268.

5. Goldie PA, Bach TM, Evans OM. Force platform measures for evaluating postural control: reliability and validity. Arch Phys Med Rehabil. 1989;70:510-517.

6. Olmsted LC, Hertel J. Influence of foot type and orthotics on static and dynamic postural control. J Sport Rehabil. 2004;13:54-66.

7. Gribble PA, Hertel J. Effect of lower-extremity muscle fatigue on postural control. Arch Phys Med Rehabil. 2004;85(4):589-592.

8. Gribble PA, Hertel J, Denegar CR, Buckley WE. The effects of fatigue and chronic ankle instability on dynamic postural control. J Athl Train. 2004;39(4):321-329.

9. Kinzey SJ, Armstrong CW. The reliability of the star-excursion test in assessing dynamic balance. J Orthop Sports Phys Ther. 1998;27(5):356-360.

10. Hertel J. Functional instability following lateral ankle sprain. Sports Med. 2000;29(5):359-369.

11. Earl JE, Hertel J. Lower-extremity muscle activation during the star excursion balance tests. J Sport Rehabil. 2001;10:93-104.

12. Hertel J, Miller SJ, Denegar CR. Intratester and intertester reliability during the star excursion balance tests. J Sport Rehabil. 2000;9:104-116.

13. Cote KP, Brunet ME, Gansneder BM, Shultz SJ. Effects of pronated and supinated foot postures on static and dynamic postural stability. J Athl Train. 2005;40(1):41-46.

14. Susco TM, Valovich McLeod TC, Gansneder BM, Shultz SJ. Balance recovers within 20 minutes after exertion as measured by the balance error scoring system. $J$ Athl Train. 2004;39(3):241-246. 
15. Wilkins JC, Valovich McLeod TC, Perrin DH, Gansneder BM. Performance on the balance error scoring system decreases after fatigue. J Athl Train. 2004;39(2):156-161.

16. Seliga R, Bhattacharya A, Succop P, Wixhstrom R, Smith D, Willeke K. Effect of work load and respirator wear on postural stability, heart rate, and perceived exertion. Am Ind Hyg Assoc J. 1991;52:417-422.

17. Robertson RJ, Moyna NM, Sward KL, Mikkich NB, Gross FL, Thompson PD. Gender comparison of RPE at absolute and relative physiological criteria. Med Sci Sports Exerc. 2000;32:2120-2129.

18. Caron O. Effects of local fatigue of the lower limbs on postural control and postural stability in standing posture. Neuroscience Letters. 2003;340:83-86.

19. Huston JL, Sandrey MA, Lively MW, Kotsko K. The effects of calf-muscle fatigue on sagittal-plane joint-position sense in the ankle. J Sport Rehabil. 2005;14:168-184.

20. Vuillerme N, Forestier N, Nougier V. Attentional demands and postural sway: the effect of the calf muscles fatigue. Med Sci Sports Exerc. 2002;34(12):1907-1912.

21. Hertel JN, Guskiewicz KM, Kahler DM, Perrin DH. Effect of lateral ankle joint anesthesia on center of balance, postural sway, and joint position sense. J Sport Rehabil. 1996;5:111-119.

22. Forestier N, Teasdale N, Nougier V. Alteration of the position sense at the ankle induced by muscular fatigue in humans. Med Sci Sport and Exerc. 2002;34(1):117122.

23. Yaggie JA, McGregor SJ. Effects of isokinetic ankle fatigue on the maintenance of balance and postural limits. Arch Phys Med Rehabil. 2002;83:224-8.

24. Wilkstrom EA, Powers ME, Tillman MD. Dynamic stabilization time after isokinetic and functional fatigue. J Athl Train. 2004;39(3):247-253.

25. Corbeil P, Blouin JS, Begin F, Nougier V, Teasdale N. Perturbation of the postural control system induced by muscular fatigue. Gait Posture. 2003;18:92-100.

26. Vuillerme N, Nougier V, J Prier J. Can vision compensate for a lower limbs muscular fatigue for controlling posture in humans. Neuroscience Letters. 2001;308:103-1

27. Patikas D, Michailidis C, Bassa H, Kotzamanidis C, Tokmakidis S, Alexiou S, Koceja DM. Electromyographic changes of agonist and antagonist calf muscles during maximum isometric induced fatigue. Int J Sports Med. 2002;23:285-289. 
28. Kawakami Y, Amemiya K, Kanehisa H, Ikegawa S, Fukunaga T. Fatigue responses of human triceps surae muscles during repetitive maximal isometric contractions. J Appl Physiol. 2000;88:19169-1975.

29. Lepers R, Maffiuletti N, Rochette L, Brugniaux J, Millet F. Neuromuscular fatigue during a long-duration cycling exercise. J Appl Physiol. 2002;92:1487-1493.

30. Mahon AD, Duncan GE, Howe CA, Del Corral P. Blood lactate and perceived exertion relative to ventilatory threshold: boys versus men. Med Sci Sports Exerc.

1997;29:1332-1337.

31. van Deursen RWM, Cavanagh PR, van Ingen-Schenau G, Becker MB, Ulbrecht JS. The role of cutaneous information in a contract control task of the leg in humans. Hum Mov Sci. 1998;17:95-120.

32. Caron O. Is there interaction between vision and local fatigue of the lower limbs on postural control and postural stability in human posture? Neuroscience Letters. 2004;363:18-21.

33. Hiemstra LA, Lo IK, Fowler PJ. Effect of fatigue on knee proprioception: implications for dynamic stabilization. J Orthop Sports Phys Ther. 2001;31(10):598-605.

34. Garland SJ. Role of small diameter afferents in reflex inhibition during human muscle fatigue. J of Physiol. 1991;435:547-558.

35. Gandevia SC. Neural control in human muscle fatigue: changes in muscle afferents, moto neurons and moto cortical drive. Acta Physiol Scand. 1998;162:275-283.

36. Li KC, Ingersoll CD, Cordova ML, Edwards JE. Soleus h-reflex diminishes following fatiguing exercises.(Abstract) J Athl Train. 2002;37(2):S-41.

37. Fitts RH. Selected papers from the third IOC world congress on sport sciences. Muscle fatigue: the cellular aspects. Am J Sports Med. 1996;24(6).

38. Skinner HB, Wyatt MP, Hodgdon JA, Conrad DW, Barrack RL. Effect of fatigue on joint position sense of the knee. J Orthop Res. 1986;4:112-118. 
APPENDICES 


\section{APPENDIX A}

\section{THE PROBLEM}

\section{Research Question}

It appears that every year new studies are published examining types of injuries, different methods of rehabilitation, as well as a plethora of other physiologic topics related to athletic training and sports medicine. However, in the past year, few studies have been published looking at time of injury with regard to fatigue. From my current experience working with a NCAA Division I Women's Basketball program I have observed that 76\% (35 of 46 total documented injuries for two calendar years) occurred during the second half of competition, or during the second half of practice. These injuries ranged from first degree lateral ankle sprains, to four complete ACL ruptures. Although two years is not an extensive time period to show a considerable trend, it seems that these injuries may have been related to athletes becoming fatigued during a competition or practice.

Most current studies ${ }^{19,20,39,40}$ examining fatigue and lower extremity injury is limited to the combination of fatigue and postural sway or joint position sense. However, little research has been completed on the relationship between joint position sense at the ankle and knee following various fatiguing exercises; in addition these studies have all neglected to comment substantially on the effects of fatigue on functional or dynamic postural control. ${ }^{7,18,19,20,39}$

In a recent study Gribble and $\mathrm{Hertel}^{7}$ evaluated at the effects of local muscle fatigue on static postural control as measured by a 30 second unilateral stance test. Through their research they were able to conclude that there is an effect on the lower extremity sagittal plane movers when subjected to local fatigue. This coincides with Miller and Bird ${ }^{41}$ who 
concluded in 1976, that fatigue to the proximal muscles adversely affected static postural control more than fatigue applied to the more distal muscles of the lower extremity. ${ }^{7,41}$ Through their research, Gribble and Hertel ${ }^{7}$ were also able to conclude that, "Slowed conduction of afferent signal from the fatigue altered state of the muscle may lead to slowed propagation of efferent signals to help maintain posture." From this information they were able to theorize that the fatigued individual will be at a greater risk for musculoskeletal injury and therefore steps need to be taken during strength and conditioning and rehabilitation to combat fatigue. ${ }^{7}$

More specifically there is limited research available examining a sport-related fatiguing process and the effects it would have on the lower quarter and core as it relates to postural stability. ${ }^{18,42} \mathrm{~A}$ sport related fatiguing process would involve an exercise bout that would more closely resemble a "typical" practice or game and would fatigue the entire body instead of just concentrating on a single muscle or muscle group. ${ }^{14,15}$ This fatiguing process would include various types of exercise in an attempt to fatigue the athlete including but not limited to isokinetic exercise, isotonic exercise, plyometric and agility exercises, as well as testing their cardiovascular endurance. ${ }^{15}$

It seems with the lack of current research linking dynamic postural control and fatigue, and the current concepts in rehabilitation that one would want to examine the possible connection between overall body fatigue and how it interacts with dynamic postural control. Therefore the question that this study poses is how dynamic or functional postural control as measured by the Star Excursion Balance Test, is affected by a sport related functional fatiguing protocol. 
Experimental Hypotheses

1. There will be a significant decrease between pre and post fatigue scores for the anterior excursion using the Star Excursion Balance Test.

2. There will be a significant decrease between pre and post fatigue scores for the anteriolateral excursion using the Star Excursion Balance Test.

3. There will be a significant decrease between pre and post fatigue scores for the lateral excursion using the Star Excursion Balance Test.

4. There will be a significant decrease between pre and post fatigue scores for the posterolateral excursion using the Star Excursion Balance Test.

5. There will be a significant decrease between pre and post fatigue scores for the posterior excursion using the Star Excursion Balance Test.

6. There will be a significant decrease between pre and post fatigue scores for the posteromedial excursion using the Star Excursion Balance Test.

7. There will be a significant decrease between pre and post fatigue scores for the medial excursion using the Star Excursion Balance Test.

8. There will be a significant decrease between pre and post fatigue scores for the anteromedial excursion using the Star Excursion Balance Test.

9. There will be a significant decrease between pre-test to mid-test to post-test on the RPE.

Assumptions

1. All subjects will meet the inclusion criteria (healthy and physically active - exercise at least $3 \mathrm{x}$ week for 30 minutes) and not the exclusion criteria.

2. The number of female participants will be approximately equal to the number of male participants based on inclusion and exclusion criteria following a random sampling process.

3. The subjects will perform the fatiguing protocol and Star Excursion Balance Test to the best of their ability.

4. The Star Excursion Balance Test is a sensitive, reliable, and valid test of dynamic postural control.

5. The principal investigator will be reliable in recording measurements for the Star Excursion Balance Test 
6. The fatiguing procedure used is representative of fatigue evident in sports participation.

7. Rating of Perceived Exertion is a valid and reliable instrument to measure perceived exertion.

Delimitations

1. Only college aged healthy subjects were used, therefore, this study can not be generalized to the rest of the population.

2. Only the Star Excursion Balance Test was used to measure dynamic balance.

\section{Operational Definitions}

1. Afferent Division - "The portion of the peripheral nervous system that carries information from the periphery to the central nervous system." $43-45$

2. Balance - The process of maintaining a center of gravity within the body's own base of support. ${ }^{39}$

3. Central Fatigue - The reduced recruitment of new motor units or decreased firing frequency of the active units, or both. ${ }^{46}$

4. Dominant Leg - The leg the subject would use to kick a soccer ball.

5. Dynamic Postural Control - "Completion of a functional task without compromising one's base of support." 2,3,44,45

6. Efferent Division - "The portion of the peripheral nervous system that carries instructions from the central nervous system to the effector organs." $43-45$

7. Functional Reach - "Reaching of a limb while challenging an individual's limit of stability." 6

8. Golgi Tendon Organ - "Receptors found at the junction of the tendons and muscle fibers that respond to both stretch and contraction of the muscle." $43-45$

9. Joint Position Sense - The body's conscious awareness of a joints position and/or movement resulting from the proprioceptive input to the central nervous system. ${ }^{21}$

10. Kinesthesia - "A specialized variation of the sensory modality of touch that encompasses the sensation of joint movement." $44,45,47$

11. Mechanoreceptor - "A sensory receptor sensitive to mechanical energy, such as stretching or bending." $43-45$ 
12. Muscle Fatigue - "A reduction in the force-generating capacity regardless of the task performed and after repeated muscle contractions" 7,25

13. Muscle Spindle - "Muscle receptors that send information about muscle length and rate of change in length." $43-45$

14. Neuromuscular Fatigue - "Any exercise-induced reduction in maximal voluntary force." 29

15. Peripheral Fatigue - Results from a decrease in the efficiency of the contractile units of the individual muscle fibers. ${ }^{17,20}$

16. Proprioception - The unconscious awareness of movement and spatial orientation arising from stimuli within the body itself. ${ }^{13,19,39}$

17. Ruffini Corpuscles - Specialized nerve endings in the skin that are sensitive to touch, tension, and possibly heat. When located in joint capsules and ligaments, they are sensitive to changes in position. ${ }^{44,45,47}$

18. Star Excursion Balance Tests - "A series of unilateral minisquats performed while attempting with the opposite leg to reach as far as possible in a given direction." (Anterior, Anterolateral, Lateral, Posterolateral, Posterior, Posteromedial, Medial, Anteromedial) ${ }^{9,11}$

19. Static Postural Control - "Maintaining a stationary position while standing on either one or both feet." 2,3

\section{Limitations}

1. Limitations to this study include a population sample consisting of a limited age range (18-28 years of age).

2. This study was a subjective measure of fatigue rather that blood metabolite markers.

Significance of the study

The relationship between fatigue and dynamic postural control may have practical significance to the fields of athletic training and or strength and conditioning. Implications of fatigue could help determine if changes are needed in an athlete's strength and conditioning programs to help combat the effects of early fatigue and thus maximize the 
benefits of strength and endurance training. With the current trends for postural training in strength and conditioning indicating that when an athlete is fatigued their postural stability is negatively impacted, may lead to re-examining of the goals of strength training.

Hopefully these new findings would help postural control training become as common as the squat, bench press, and power clean in the weight room.

Examining further the impact of fatigue on injury may shed some light on ways that athletic trainers can help prevent athletic injuries, thus allowing the athletes to practice and play under optimal physical conditions. This new research may allow athletic trainers to do preseason screening of postural control thus finding the athletes who need to work on this aspect of their conditioning. Early identification of those athletes would help the strength and conditioning staff target this area in those athletes, and would hopefully lead to a decrease in injuries due to weakness or lack of conditioning leading to fatigue. These preseason screenings may be similar to those developed by Colorado College in their attempt to screen athletes for potential ACL injuries. Although not evaluated in this study, research examining the impact of fatigue on postural control and core stability and how that relates to injuries to the different body regions should be explored.

Since we know that decreased dynamic or functional postural control can lead to injuries especially with females and ACL tears, it would be helpful to identify if fatigue plays a role here. Another factor to consider is that the proprioceptive muscles are smaller than the major muscles that move to the joints of the body, especially around the knee. If you get into a fatigue situation where you have decreased cross bridge formation, or cross bridges that stay in the contracted formation, a smaller muscle will fatigue more rapidly than a larger muscle that has more muscle fibers, and thus more cross bridges to spare. 
The link between injury rehabilitation and dynamic postural control has already sparked new research in the area. If fatigue is shown to negatively impact dynamic postural control, then this data may be helpful in the development of new rehabilitation protocols much like the ones presented by Colorado College. ${ }^{48}$ Knowing that fatigue might lead to poor postural control and perhaps impaired core stability can allow a clinician to emphasize these exercises in rehabilitation in order to help condition this area. In addition to emphasizing dynamic postural control in rehabilitation, it would also help to work on muscular endurance. As previously mentioned, the muscles most responsible for proprioception are smaller and will often fatigue more rapidly, thus by including an endurance program will help combat the effects of fatigue, and potentially increase dynamic postural control.

These connections between fatigue and postural control may also shed some light into length of practices or make-up of practices. Most coaches do not approach the athletic training staff at the beginning of each season asking for advice on length of practice or optimum time of practice. However, if fatigue indeed plays a large role in decreasing overall postural stability and thus increases the likelihood of injury, the athletic trainer can present this information to the coach so that they may make an informed decision about length and or make-up of practice.

If through this study we are able to observe that fatigue negatively affects dynamic postural control then it could have implications on all of the aforementioned points. All professionals involved with athletes should work together as a team to provide better quality care for the athlete. Coaches would take this information and make informed decisions when establishing practice times as well as length of practices. Strength and 
conditioning coaches could design programs around decreasing the effects of fatigue and training the postural control system. Athletic trainers could incorporate prevention programs by adding postural control strategies in all facets of rehabilitation. 


\section{APPENDIX B}

\section{LITERATURE REVIEW}

Introduction

Until recently, most research completed on proprioception and the lower extremity examined balance or joint position sense. These studies often examined proprioception of the ankle and/or knee during an injured state, or evaluated the benefits of balance training when rehabilitating an injury. More recently, fatigue has become a popular focus of research, examining fatigue and the relationship to ankle sprains $4,7,8,10,19,21,22,49$ and knee injuries ${ }^{33,38,50}$ with regard to altered postural control. In 2004, Gribble and Hertel ${ }^{7}$ examined this further as they conducted a study on how fatiguing various muscles of the lower extremity (flexors and extensors of the hip and knee, and dorsiflexors and plantarflexors of subtalar joint) affected static postural stability as measured by a static unilateral leg stance. ${ }^{7}$ They found that fatiguing muscles more proximally altered static postural control more than fatiguing the more distal muscles. ${ }^{8}$

However as we know, most sporting activities rely on dynamic motions that involve a coordinated effort from many major agonist muscles as well as the support of those smaller proprioceptive muscles. An attempt has been made to examine dynamic postural stability as it relates to movement as well as injury. On the other hand, there is little to any research available on the affects of fatigue on dynamic postural control. One would postulate that if large muscles like the gluteus maximus, and the rectus femoris are used to perform in a sport, then the much smaller muscles that provide proprioceptive feedback kinesthetically would fatigue sooner. As defined in the literature, dynamic postural control is the "completion of a functional task without compromising one's base 
of support." $" 2$ This is important in any sport such as batting in baseball, hitting a forehand in tennis, or kicking a soccer ball. To achieve greatness in any of these activities one needs to be able to maintain dynamic postural control during coordinated movement and the positioning of the body in space which is achieved by the dynamic stabilizers in and around the joints.

With this in mind, my research will examine this relationship between activity related fatigue and dynamic postural control. The following topics will be discussed in the literature review, proprioception and the role of the muscle mechanoreceptors the articular mechanoreceptors, and the cutaneous receptors. The proprioception section will also discuss the impact of the peripheral and central nervous systems. The literature review will also examine neuromuscular control including feedback, feedforward, and postural control. From there the review will cover fatigue, and specific fatiguing protocols and the relation to postural control.

\section{Proprioception}

Many authors have defined proprioception with little agreement. Myers ${ }^{51}$ defined proprioception as "the specialized variation of the sensory modality of touch that encompasses the sensation of joint movement." Lephart ${ }^{39}$ goes further to include joint position as well as joint movement in his definition of proprioception. In order to clarify the meaning of proprioception Riemann and Lephart ${ }^{45}$ wrote a review article in 2002 that evaluated the current literature in an attempt to come up with one unified definition that could be used by all clinicians. That definition states that "proprioception describes 
afferent information arising from internal peripheral areas of the body that contribute to postural control, joint stability, and several conscious sensations."

In order to fully understand the definition of proprioception, one must first understand the different components beginning with the sensorimotor system. The sensorimotor system is a subcomponent of the bodies entire motor control system. ${ }^{39,40,45 \text {, }}$ ${ }^{49,51}$ The term was described in 1997 as the motor, sensory, and central integration and processing components that are involved in maintaining joint homeostasis during body movements. ${ }^{40,45,49}$ Within this definition, maintaining joint homeostasis during body movements was described as functional joint stability. ${ }^{45}$ In order to maintain functional joint stability a balanced relationship must be formed between the two components, the static, and dynamic. The static or passive components consist of the joint capsule, cartilage, ligaments, friction, and the bony geometry within the articulation itself ${ }^{45,52}$ The dynamic portions of functional joint stability arise from the feedforward and feedback loops of neuromotor control. These loops control the skeletal muscles that cross the joint and their effectiveness is determined by the physical characteristics of the joint including muscular strength, muscular endurance, and joint range of motion. ${ }^{39,45}$

Peripheral nervous system: The peripheral nervous system (PNS) consists of the peripheral portion of the nervous system external to the brain and spinal cord. Also known as the afferent response to a stimulus, the PNS takes all input from its receptors and relays that information back to the central nervous system (CNS) which consists of the brain and spinal cord. ${ }^{39,45,51}$ This feedback to the CNS is mediated by a series of mechanoreceptors which are primarily found in the muscle, ligament, tendon, and joint capsule. ${ }^{39,45}$ The 
mechanoreceptors found in the deep skin and fascial layers are traditionally associated with tactile sensation, and are considered secondary or supplementary mechanoreceptors. ${ }^{45}$ Mechanoreceptors: A mechanoreceptor is "a specialized neuroepithelial structure found in the skin and in articular, ligamentous, muscular, and tendinous tissues about a joint." ${ }^{51}$ In general these are specialized sensory receptors that are responsible for converting physical or mechanical events that occur in the periphery, into signals that can be understood by the CNS. In order for these receptors to work they need to receive some stimulation from the periphery. Activation of joint mechanoreceptors is either triggered by the deformation and/or loading of the soft tissues that surround the joint. ${ }^{32,39,45,52,53}$ Once stimulated, the mechanoreceptors send neural stimulation to the CNS along the afferent pathways of the peripheral nervous system. The CNS then decides how to integrate these messages via cortical and reflex pathways. ${ }^{35,39,45}$ Depending on the type, intensity or duration of the sensation, different types of mechanoreceptors may respond to the stimuli, and will demonstrate different adaptive properties depending on a particular stimulus. ${ }^{39}$

The mechanoreceptors of the body can be further separated into two groups based on their location. The first group is muscular mechanoreceptors which consist of muscle spindles, golgi tendon organs, and small-diameter muscle afferents. In their entirety, muscle spindles (Ia afferents) are responsible for sensing and conveying information pertaining to muscle length and rate of change in length, respectively. ${ }^{1,19,39,45}$ They consist of dedicated afferent nerve endings that are attached to a small bundle of modified muscle fibers called intrafusal fibers. ${ }^{1,39,45}$ These intrafusal fibers are innervated by the gammamotor neurons, where as the extrafusal fibers, which make up the bulk of the muscle which are responsible for generating force, are innervated by the alpha-motor neurons. ${ }^{39}$ The beta 
motor neurons connect to both the intrafusal and extrafusal muscle fibers. Within the intrafusal fibers there are two types of fibers, annulospiral endings and flower spray endings. The annulospiral endings are sensitive to changes in length, but less sensitive to static length. A faster stretch of muscle fibers will mean that more impulses will be sent by the annulospiral afferents (1a afferents). ${ }^{45,54}$ The flower spray endings are more sensitive to static muscle length, and not to changes in length. The more the muscle is stretched, the more impulses that are sent by the flower spray afferents (SpII afferents). ${ }^{44,45}$

When a muscle contracts, alpha-gamma co-activation is believed to be the mechanism that allows length and tension of the muscle to be monitored. ${ }^{39,45}$ Contraction of the peripheral contractile units (extrafusal fibers) leads to a stretching of the central regions that contain the sensory receptors (intrafusal fibers) on both ends. This activation of the gamma-motor neuron allows for the re-adjustment of muscle spindle sensitivity by the CNS (gamma bias) when the extrafusal fibers are shortened. This then leads to an increased rate of firing within the sensory endings and also an increase in the sensitivity of the muscle spindle to length changes. ${ }^{1,39,45}$ When a muscle is loaded beyond the anticipated level, the intrafusal fiber shortening occurs more than the extrafusal fiber shortening. This sudden stretching of the spindles in the central region causes a sudden influx of postsynaptic potentials from the spindle afferents. ${ }^{39}$ Then, with the addition of these signals to the alpha-motor neurons from the descending pathways, there is an increased force production. ${ }^{39,45}$

In addition to being loaded more than anticipated, a sudden stretch to a muscle can cause what is known as a stretch reflex. Muscle spindles are also responsible for this phenomenon. When the afferent fibers from the spindle enter the CNS at the spinal cord, 
they divide into several different paths. One path connects with an interneuron in the spinal cord and returns to directly stimulate the motor neurons going back to the muscle. When that muscle is subjected to a sudden stretch, these returning pathways excite the motor neurons, causing a sudden change in force production usually leading to an external movement, thus completing a reflex arc known as the stretch reflex, or myotatic reflex. $1,39,45$

Another type of afferent mechanoreceptor located in the muscle is the Golgi Tendon Organ (GTO) also known as the (Ib afferents). ${ }^{19}$ These receptors are located in the musculotendinous junction at varying intervals. ${ }^{45}$ The GTO is responsible for sending information regarding muscle tension or rate of change of muscle tension. ${ }^{1,39,45} \mathrm{~A}$ small bundle of muscle tendon passes through each GTO eventually attaching to muscle fibers. The series make-up and arrangement of the GTO, tendon and muscle fibers, coupled with a high dynamic sensitivity, and very low threshold allows the GTO to provide the CNS with rapid feedback with regards to muscle tension. ${ }^{1,39,45}$ Unlike the muscle spindles that detect length of the muscle, GTO's function primarily in signaling active muscle tension which is the tension developed during contraction, rather than passive tension which is tension created by inactive muscle stretching. ${ }^{1,39,45}$

Golgi tendon organs are recruited when muscle contraction pulls on the tendon, resulting in straightening of the collagen bundles and thus distorting the receptor endings of the afferent neurons. ${ }^{39}$ When this distortion occurs, there is an increase in the discharge rate of action potentials of the corresponding receptors that synapse in the spinal interneurons. ${ }^{39,45}$ to innervate the muscles that are being stretched. At the same time as the inhibition of the stretched muscles, there is an inverse action of excitation of the motor 
nerves of the antagonist muscles resulting in the opposite motion to help decrease the current stretch. ${ }^{39,45}$ Since the GTO is designed as a protective mechanism, it relaxes the muscle that is being overstretched and fires the antagonist to bring the muscle out of that overstretched state.

The last type of muscle mechanoreceptors are known as small-diameter muscle afferents (Type III and IV). These muscle afferents innervate all of the free nerve endings that are located in large numbers around the muscle. ${ }^{19}$ Often times these afferents are silent, and will respond to biomechanical, local mechanical, and thermal events. ${ }^{19}$

The second group of mechanoreceptors is known as the articular mechanoreceptors because these receptors lie in and around the joint or joint capsule. There are four kinds of articular receptors in the body. ${ }^{55}$ The first, type I have a low threshold level and slowly adapting receptors. Type I articular receptors are located in the stratum of the capsule. Type II receptors are located in the synovial membrane, fibrous layers of the joint capsule, and fat pads. The type II receptors are also low threshold like type I, but these receptors are fast-acting, unlike type I. Type III articular receptors are located in the ligaments and are high threshold, active at the end range of motion, and slow adapting. ${ }^{55}$ The type IV articular receptors are free nerve endings that are only stimulated by noxious stimuli.

The Ruffini endings act as both static and dynamic receptors based on their lowthreshold, and slow-adapting characteristics. ${ }^{45}$ These nerve endings are located deep in the skin and in the collagenous fibers of the joint capsule. Like other mechanoreceptors, they respond to mechanical tissue deformation, however their response is to a continued state of pressure or deformation. ${ }^{39,45}$ These endings will provide information on joint position changes, as well as joint position. 
The other type of articular mechanoreceptors is called Pacinian Corpuscles. Unlike Ruffini endings, these receptors are classified as purely dynamic in nature due to their lowthreshold but rapidly adapting characteristics. ${ }^{39,45}$ These onion-shaped receptors are located deep in the skin around joints, ligaments, and tendon sheaths. Unlike Ruffini endings that are sensitive to constant pressure, the Pacinian corpuscles are activated by a rapid change in joint angle that puts pressure on the corpuscle. ${ }^{39,45,54}$ These corpuscles are sensitive to the initial change in joint motion thus providing information about joint movement, and little about the current joint position.

The last types of receptors are the cutaneous receptors. Although not classified as a major receptor, these cutaneous receptors still play a vital role in proprioception. These receptors are represented by free dendritic endings that are especially plentiful in the hands and feet. ${ }^{21,45}$ The cutaneous afferents combine with the motor neurons of the hand and foot to create a full receptor loop. In fact, these receptors can be very sensitive to pressure, as "mild pressure to the fingers, can produce relatively strong contractions of digit muscles like the thenar muscles and the flexor digitorum superficialis." 54

Central nervous system: The central nervous system is made up of the brain, brain stem, and the spinal cord which acts as a link between the PNS and the central nervous system (CNS). The CNS tissue consists of two types with the first being the white matter which is made up of myelinated axons, and the gray matter which is made up of mostly neuron cell bodies and unmyelinated processes. ${ }^{45,52,54}$

To understand proprioception, it is important that we first understand the overall make-up of the CNS and its components. The brain can be divided into three different areas each with its own distinct characteristics. First is the cerebrum which is made up of 
the left and right cerebral hemispheres, cerebral cortex, and basal ganglia. ${ }^{45,54}$ In general the cerebrum acts to organize complex movements, receive sensory information and store learned experiences. The cerebrum can further be divided into superficial and deep areas. The superficial area is the cerebral cortex. This superficial outer layer of gray matter houses the areas of the conscious mind and can be divided into the Brodmann areas. ${ }^{54}$ These Brodmann areas, which are areas of the cortex that relates to functional areas of control, are divided into the sensory cortex, the association cortex, and the motor cortex. The sensory cortex is responsible for the general somatic senses, and conscious awareness. ${ }^{54}$ The association cortex integrates sensory information and is involved with higher brain functions like cognition. ${ }^{54,56}$ The motor cortex is responsible for, "initiating and controlling more complex and discrete voluntary movements." ${ }^{45}$ It can be also divided into three specialized areas, each of which project directly and indirectly via the brain stem onto the interneurons and motor neurons. ${ }^{45,54,56,57}$ The first area is the primary motor cortex. It receives information from the peripheral afferents, and is responsible for telling the muscles to be activated, how much force for them to produce, and in which direction to act. ${ }^{45,52}$ The premotor area is the second area of the motor cortex. It also receives sensory information, however, it mainly is involved in organization and preparation of the movement commands. ${ }^{45,54,57}$ The supplemental motor area is the third specialized motor area of the motor cortex. This area is important for programming complex sequences of movements that involve many different muscles or muscle groups.

In the motor cortex, pyramidal cell axons originate to form the pyramidal tract which is the major motor-controlling tract of neurons. These motoneurons will course towards the spinal cord where they will terminate making direct connections with other 
motoneurons that go out to muscles. ${ }^{54,56}$ The motor cortex plays a large role in movement, but needs substantial input from other regions such as the cerebellum, and basal ganglia, to produce coordinated movements. The motor cortex will integrate this information and then send out a movement plan via the (extra) pyramidal tracts. ${ }^{54}$

The other part of the cerebrum is the deep matter. This area houses the cerebral white matter and the communication link between the two halves of the brain, the corpus collosum. ${ }^{54}$ In this area the basal nuclei or ganglia are also housed. These are usually deep to the white matter but can also be contained within the white matter. These structures are involved with initiation, intensity, and stopping of motor outflow, and can also be involved with the learned aspects of posture and equilibrium. ${ }^{54}$

The second general region of the brain is the cerebellum. The cerebellum is involved with smooth and coordinated movement, especially for very rapid movements. The most important function is to process and compare intended movement commands from the motor cortex and incoming PNS sensory information, and to feedback the updated information to the motor cortex. ${ }^{45,54,56-58}$ The cerebellum is divided into three functional divisions. ${ }^{45,58}$ The first division receives the vestibular input, both directly and indirectly from the vestibular labyrinth (semicircular and otolith receptors) and is also involved with postural equilibrium. The second cerebellar division is mostly responsible for the initiation and planning of movements, especially those requiring precise and rapid limb movements. The third division, the spinocerebellum, receives the somatosensory information conveyed through the four ascending spinocerebellar tracts. As well as using somatosensory input, the third division also receives input from the vestibular labyrinth, visual, and auditory organs. ${ }^{45}$ This output serves to adjust ongoing movements through 
connections on the medial and lateral descending tracts in the cortex and brain stem. ${ }^{45,58}$ The third division uses the somatosensory input as a feedback regulation of muscle tone by regulating the static gamma motorneuron drive to the specific muscle spindles.

Another associated area of this general section of the brain is the basal ganglia. These consist of five subcortical nuclei, "located deep within the cerebral hemispheres." 45 Unlike the cerebellum which has input and output connections with all three levels of motor control, the basal ganglia has input and output connections via the thalamus to the cerebral cortex. The basal ganglia are involved with receiving input from the entire cerebral cortex, and are involved in the higher-level, cognitive aspects of motor control. ${ }^{45}$

The third general area of the brain is the brain stem which includes the medulla oblongata, the pons, and the midbrain. This area is mostly used for "programmed, automatic movement behavior" like "locomotion, and posture." ${ }^{54}$ This area contains many clusters of neuron cell bodies that act as a passageway for all fibers passing between the spinal cord and the cerebrum. This area is also responsible for receiving and integrating information from all regions of the CNS through a complex neural network. ${ }^{54}$

The other portion of the central nervous system is the spinal cord. This is the true link between the CNS and the PNS. In fact, many scientists believe that integration of all sensory input that is received from the body begins at the spinal cord. "Integration describes the summation, gating, and modulation mechanisms that occur as a result of various combinations of excitatory and inhibitory synapses with the afferent neurons." 45 This afferent integration is an essential part of coordinated movement, and smooth motor control, and occurs at all levels of the CNS. ${ }^{39,45,59}$ 
Most proprioceptive information travels to the higher levels of the CNS (i.e. the brain) through either the dorsal lateral tracts, or the spinocerebellar tracts. ${ }^{45}$ There are two dorsal lateral tracts, and they are located in the posterior region of the spinal cord and will end up relaying messages to the somatosensory cortex. The majority of these signals are pressure, vibration, and touch, however some conscious awareness of position and "kinesthetic sensations" ${ }^{45}$ is attributed to the dorsal lateral tracts. On the other hand, the spinocerebellar tracts are characterized by the fastest transmission velocities in the CNS. This tract ends in the cerebellum where signals can be processed and integrated with other afferent information and descending information. ${ }^{45}$ Unlike the dorsal lateral tracts, the spinocerebellar tracts transmit nonconscious proprioception, which is limb position, muscle length, joint angles, and muscle tension. ${ }^{45}$ This information is mostly used for automatic, reflex, and voluntary movements.

A general model of voluntary movement would sound something like this. The cortical or subcortical areas generate a will to move. This information is used by the association cortex, basal ganglia, and cerebellum to gather a movement plan and then refine it. The plan is then relayed through the motor cortex, using the thalamus, and down the pyramidal and extra-pyramidal tracts to the spinal motor neurons. The motor neurons then send out the message to contract the muscle through efferent control of the muscles. Along the way, there is feedback at each level that is used to refine the movement, and to assure that one accomplishes what they set out to do. ${ }^{39,45,54,59,60}$

\section{Neuromuscular Control}

This term, neuromuscular control is a term that is often used in the literature to describe many different aspects of control by the nervous system. Because, of its broad 
nature, the term neuromuscular control can refer to all aspects of the nervous system control over muscle activation, integration, and all factors contributing to performance on a task. ${ }^{45}$ If simply looking from a joint stability perspective, Riemann and Lephart ${ }^{45}$ defined neuromuscular control as "the unconscious activation of dynamic restraints occurring in preparation for and in response to joint motion and loading for the purpose of maintaining and restoring joint stability.” Even simpler, neuromuscular control is the efferent response to the afferent information that is received by the numerous mechanoreceptors mentioned earlier. The response from the CNS will be a reflex like mentioned in the muscle spindle section where the muscle spindle senses a stretch to the muscles and sends afferent information to the spinal cord. In the spinal cord, it synapses with an interneuron which then connects to the efferent motor neuron and causes the "knee-jerk" reaction. ${ }^{45,52,54}$ The best example of neuromuscular control in the body is how the small muscles we sometimes call proprioceptors, react to forced inversion of the ankle unconsciously in an attempt to evert the ankle.

A major part of neuromuscular control is the feedback and feedforward loops that are created as a "checks and balances" system. ${ }^{45}$ Feedback control is a stimulation of a correcting response within the remedial system in response to sensory detection. These initiated feed back actions are mostly shaped by previous experiences with the stimulus. Feedforward is described as anticipatory actions occurring before the sensory detection of a stimulus. Both types of control use somatosensory, visual, and vestibular input to provide the information necessary for motor control, but the methods of processing the afferent signals are different. Feedback control uses a continual processing of afferent information that provides a response control on a more "moment-to-moment basis." ${ }^{45}$ However, 
feedforward uses the afferent information intermittently until the feedback controls are initiated.

The real problem comes when trying to classify an action as either feedforward or feedback. Unlike their definitions, in some circumstances a combination of both controls exists as in the maintenance of postural control. ${ }^{45}$ For example, consider the situation in which a subject watches a tester trigger a device that induces a joint movement. Many subjects will naturally tense up when they see the tester beginning to push the trigger before movement. There is speculation on whether the muscle activation before the joint perturbation is a result of feedforward or feedback control. ${ }^{39,45}$ Because of this, feedforward control should describe the actions occurring upon identification of the beginning in addition to the effects of impending stimulus. Feedback control should then be thought of as "the actions occurring in response to the sensory detection of direct effects from the arrival of the event or stimulus to the system." $39,45,54$

Postural control: Measurement of postural control is very important in assessing all populations from pediatric, to geriatric, to the athletic population when trying to establish levels of neuromuscular functions. ${ }^{2}$ The postural control system operates as a feedback control between the brain and musculoskeletal system. The body uses afferent information obtained from the mechanoreceptors in the feet, legs and trunk, and uses the feedback loop to maintain posture and allow the individual to stand erect against the effects of balance. 1,4,45 However this is not all that affects postural stability, it is also influenced by nervous system disorders, dysfunction of the optic nerve, mental stress, vestibular mechanisms, and also fatigue. ${ }^{1}$ The body uses feedback obtained from the vestibular, visual, and 
proprioceptive sensors and relays commands to the muscles in the extremities which will then generate enough force to maintain the upright posture.

When thinking about equilibrium, the organ most responsible for detecting sensations is the vestibular apparatus. This is composed of a series of bony tubes and chambers within the temporal bone called the bony labyrinth. Information from the vestibular apparatus is used in three different ways to help maintain postural control. First this information is used to control eye movement so when the head is turned, the eyes may stay "fixed" on any one point. ${ }^{1,2}$ Second, vestibular information is used in the maintenance of posture. Third, the vestibular information can be useful for conscious awareness of body position and acceleration after this information has been relayed to the cortex through the thalamus.

The vestibular system plays a large role in stabilizing body sway, and is achieved by low levels of leg activation. ${ }^{1,4}$ Under normal conditions balance is maintained by use of somatosensory and visual inputs. Once a sudden change in movement or a perturbation is added causing the person to change the direction of movement or head position, automatic control provided by the vestibular system becomes crucial for stabilization of equilibrium.

Postural control can be described as either static (maintaining a position with minimal movement), semi-dynamic (maintaining a position while the base of support is moved) dynamic (maintaining a stable base of support while completing a prescribed movement). ${ }^{1,2,4-6}$ Static postural control is usually quantified through measurements of ground reaction forces. These types of forces are usually measured by a force plate and look at velocity, or variability. ${ }^{2}$ Some other non-instrumented methods of measuring static postural control include having an individual stand on one or both feet while attempting to 
maintain a stationary position. The most often used is the modified Rhomberg test. This test involves standing as motionless as possible and then adding demands such as closing the eyes, tilting the head back, and touching the index finger to the nose. Although this test is typically used as an assessment tool for cerebral concussions (bilateral stance) and for injury assessment (unilateral stance), it does not place movement or strength demands on the participant. ${ }^{2}$

Semi-dynamic balance involves maintaining balance while the force plate or ground surface is moved. This form of postural control is usually measured by the Biodex Stability System (BSS), or the Chattex Balance System. ${ }^{2,6}$ To measure this the subject stands on the force plate of the balance system they are using. The force plate is then moved and how the body keeps its balance during the movement is recorded as semidynamic balance. This type of balance can be useful to study because it can mimic the effects of a non-smooth playing surface, and also can be helpful in seeing how the body reacts to a slippery or muddy field when the athlete's footing may be a problem. ${ }^{5}$

Dynamic postural control on the other hand often involves the completion of a functional task without compromising the base of support. ${ }^{2}$ In addition to assessing balance, dynamic postural control adds additional demands of proprioception, range of motion, and strength while attempting to maintain an upright and steady posture. ${ }^{2}$ There are a few tests that have been developed to assess dynamic posture in the pediatric population and in the geriatric population, however there is only one test in the current literature that truly stresses the dynamic balance capabilities of healthy individuals, and that test is the Star Excursion Balance Test (SEBT). ${ }^{2,9-11,61}$ This test is said to provide a 
significant challenge to the athlete's postural control system and shows strong intra-rater reliability, and is sensitive for the screening of musculoskeletal injuries. ${ }^{2,9-11}$

Currently there are four major factors that affect postural control. Injury, chronic ankle instability, foot biomechanics, and fatigue all have been shown to alter postural control. Lateral ankle sprains are one of the most common injuries in athletics. ${ }^{21}$ Although debate exists on whether the functional instability that results is due to mechanical instability or proprioceptive deficits, it is clear that injury to the ankle ${ }^{21}$ and other areas of the lower extremity can affect postural control. ${ }^{33,38}$ With injury there is damage to the proprioceptors during an injury because the joint receptors have less tensile strength than the ligaments. ${ }^{21}$ The dysfunction that results from this injury leads to impaired postural control. ${ }^{21}$ This impaired control was evident when as anesthetic was administered to the lateral ankle joint. They concluded that inhibition of the joint receptor afferent fibers adversely affected joint proprioception assessed in weight bearing subjects. ${ }^{21}$

Another factor affecting postural control is chronic ankle instability (CAI). As mentioned above, the lateral ankle sprain is one of the most common injuries in athletics, and the chance of re-injury can be as high as $80 \%{ }^{8,21}$ Chronic ankle instability is defined in the literature as altered mechanical joint stability due to repeated disruptions to the ankle's integrity with perceived or observed deficits in neuromuscular control. ${ }^{4,6,8}$ In 2004 Gribble et al. ${ }^{8}$ found that subjects with CAI had significantly smaller reach distances when compared to the uninjured side, and a healthy control group. Nakagawa and Hoffman ${ }^{4}$ found that recurrent ankle sprains may be associated with reduced postural control in the static and dynamic conditions as evident by greater center of pressure excursions (static postural control) and smaller reach distances (dynamic postural control) on patients with 
CAI. Olmsted et al. ${ }^{61}$ obtained similar data to the two previous authors and concluded that the groups with CAI had significantly smaller reach distances as compared to the same limb in a healthy population, as well as compared to the healthy contralateral extremity.

Recently two studies have been published examining the effect of foot type on postural control. Olmsted and Hertel ${ }^{6}$ suggested that static postural control might not be sensitive enough to detect deficits in impaired subjects, because it allows them to use alternate motor strategies to accomplish the task to an adequate level even in the presence of pathology. In their study they found that after two weeks of orthotic treatment for at least four hours a day, patients with cavus feet had increased reach distances at a greater magnitude than the rectus or planus patients. Further they discovered that while wearing the orthotics, cavus patients had greater reach distances in the anterolateral, lateral, and posterolateral planes as compared to the rectus and planus groups. ${ }^{6}$ They theorized that when in normal stance patients with cavus feet have little contact with the floor by the plantar surface of the feet. They suggested that custom-fitted orthotics may enhance plantar cutaneous receptor activity by providing altered distribution of pressure along the plantar surface of the foot.

Cote et. al. ${ }^{13}$ examined the effects of the pronated and supinated foot on static and dynamic postural stability. They suggested that an excessively supinated foot characterized by a high arch and hypomobile midfoot, may not adapt to the underlying surface, increasing the demand on the surrounding musculoskeletal structures to maintain postural stability. On the other hand, a foot with excessive pronation, which is characterized by a hypermobile midfoot and a flattening of the medial arch, may place huge demands on the neuromuscular system to stabilize the foot and maintain an upright posture. When they 
examined dynamic balance and foot type with the SEBT, they found that supinators reached further in the lateral and posterolateral directions. A supinated foot would place greater amounts of pressure on the lateral aspect of the foot, therefore leading to a greater reach distance in the lateral direction. On the other hand, pronators reached further in the anterior direction than both supinators and neutrals, and further than neutrals in the anteromedial direction. This again can be explained by the nature of the deformity. Pronators tend to collapse down on the medial aspect of the foot and therefore have a reduced ability to maintain a rigid support system during full weight bearing. Because of the medial foot deviation and the greater midfoot mobility, it seems logical that they would have these differences in reach.

Fatigue

Fatigue is a very complex phenomenon that is not entirely understood. Fatigue is defined as "a reduction in the force-generating capacity regardless of the task performed." 18,25,32,35,36,62 Fatigue results from an interruption in the chain of events from the CNS to the muscle fiber. ${ }^{63}$ Although all of these authors seem to agree on the basic definition, there is still a lot of debate on whether fatigue is purely metabolic, or purely neuromuscular related, or a combination of both.

Local fatigue, sometimes called peripheral fatigue can involve, "the neuromuscular junction, the process of excitation-contraction coupling, which involves the activation of the surface membrane, the propagation of that activation down the t-tubules, the release of calcium, and the activation of the contractile elements involved in the generation of force and power. ${ }^{37}$ Peripheral fatigue is also the result of activation of "nociceptors by metabolic products of muscular contraction that include bradykinin, arachidonic acid and 
prostaglandin $\mathrm{E}_{2}$, potassium, and lactic acid." ${ }^{22}$ These metabolites along with inflammatory substances will have a direct impact on the discharge patterns of the muscle spindles. ${ }^{22}$ In addition to these metabolites it has been shown that efferent and afferent neuromuscular pathways are changed via reflexes originating from the small-diameter muscle afferents (mostly group III and group IV afferents.) ${ }^{22,34}$ Fatigue of a single muscle (myofibrillar fatigue) can be caused by changes in force production due to alternations of intracellular $\mathrm{pH}$ (acidosis), factors affecting the release of $\mathrm{Ca}^{2+}$ from the sarcoplasmic reticulum, or from the metabolites mentioned above. ${ }^{64}$

A more concise look at peripheral fatigue is presented by McArdle et al.$^{63}$ They present three situations that explain all of what was just mentioned above in a logical order. First they suggest that significant reduction in the glycogen content of active muscles can relate to fatigue in both endurance trained and untrained subjects during any prolonged high-intensity bout of exercise. ${ }^{22,37,63}$ This is what they refer to as nutrient fatigue. This will occur even when sufficient oxygen is available to generate energy through aerobic pathways. "Depletion of phosphocreatine (PCr) and decline in the total adenine nucleotide pool (ATP + ADP +AMP) that reflects a mismatch between ATP supply and demand (decreased ATP synthesis and/or increased ATP degradation) also accompanies the fatigue state in prolonged submaximal exercise in untrained individuals." 37,63

Next McArdle et al. ${ }^{63}$ goes on to elaborate on metabolites. They say that oxygen lack coupled with increased levels of blood and muscle lactate will relate to fatigue in any short-term, maximal exercise. The dramatic increase in $\mathrm{H}^{+}$concentration in the muscles which accompanies lactate accumulation, dramatically disrupts the intracellular 
environment. Fatigue can also occur at the neuromotor junctions when an action potential fails to cross from the motor neuron to the muscle fiber. However, the precise mechanism of neural fatigue is largely unknown. ${ }^{37,63}$

Central or general fatigue relates to events of neural input to the higher brain center, central command, recruitment of the alpha motor nerve pool, and the alpha motor nerves themselves. ${ }^{37} \mathrm{Fitts}^{37}$ states that central fatigue does exist, but the primary causes of fatigue in athletes seems to be peripheral in nature. Increased perceived effort, which is a signal that requires the motor cortex, will reflect the need to recruit more motor neurons and muscles, and thus the need to drive them harder. ${ }^{35}$ It seems that the $\mathrm{H}$ reflex also may play some role in central fatigue. Li et al. ${ }^{36}$ examined the soleus-H-Reflex and how it diminishes following a fatiguing exercise. They defined the H-reflex as the amount of available motoneuron pool excitability. Their study used isokinetic fatiguing of the ankle plantarflexors at $50^{\circ}$ per second. They found that muscle fatigue did indeed decrease motoneuron pool excitability. ${ }^{35}$ They went on to mention that the ability to recruit motoneurons during a voluntary isometric contraction would be decreased following a fatiguing exercise. ${ }^{36}$ McArdle et al. ${ }^{63}$ discussed central fatigue in reaction to the neurotransmitters. Exercise induced alterations in the levels of CNS neurotransmitters such as serotonin, 5-hydroxttryptamine (5-HT), dopamine, and acetylcholine in various brain regions, along with the neuromodulators ammonia and cytokines secreted by immune cells will probably modify one's psychic or perceptual state to cause decline in the ability to exercise. 
Fatiguing Protocols and Postural Control

There are many different techniques to fatigue the body, and even more specifically the lower extremity. Several studies have been completed using isokinetic contractions, isometric contractions, repetitive concentric/eccentric contractions, and functional activities to produce fatigue. However, not all of these are practical or similar to fatigue evident in athletic events. Most athletes, power lifters aside, experience a functional fatigue while playing their sport as well as central and peripheral fatigue, to the extent that they are exhausted after a game or practice. We know that performing eccentric contractions over and over again will fatigue the muscle, we know that holding an isometric contraction of the gastrocnemius and soleus will fatigue the plantarflexors. However, we know very little about how functional fatigue affects the body.

We will begin the review of the literature with isokinetic fatiguing protocols. Information on specific fatiguing protocols can be found in Table B1 as well as results and conclusions. Isokinetics is a contraction at a constant speed using the Biodex system or other brands of dynomometers. Gribble et al. ${ }^{8}$ looked at the effects of fatigue and chronic ankle instability on dynamic postural control. They had all of the subjects complete five testing sessions in which saggital-plane kinematics and reaching distances were recorded while they performed the anterior, medial and posterior excursions of the Star Excursion Balance Test. Their fatiguing protocol consisted of five maximal trials at $60 \%$ for each of the designated movement patterns (plantarflexion/dorsiflexion, flexion/extension of the knee, flexion/extension of the hip). After two minutes of rest they repeated the movement at $60 \%$ continuously until force production dropped below $50 \%$ of peak torque. They found that the involved side of the chronic ankle instability subjects had a significantly 
smaller reach distance and knee flexion angles for all three directions. They also found that the fatiguing protocols decreases reach distance and knee flexion angles further.

Another study that used an isokinetic fatiguing protocol was Yaggie and McGregor's ${ }^{23}$ study. They looked to quantify the changes in balance parameters and ranges of postural control following isokinetic fatigue of the ankle. The subjects performed one circuit of the following exercises at 100\% maximal effort: inversion, eversion, dorsiflexion, and plantarflexion. The movements were performed in the concentric/eccentric mode with a speed of $60^{\circ}$ per second. When three consecutive repetitions below 50\% maximum joint torque were performed, fatigue was assumed and they moved to the next exercise. ${ }^{21}$ Their results indicated that isokinetic fatigue of the ankle plantarflexors and dorsiflexors significantly $(\mathrm{p}<.05)$ influenced sway parameters and ranges of postural control in healthy young men.

Three other authors have used isokinetic fatigue and have reported similar results. Johnston et al ${ }^{65}$ used isokinetic fatigue on the ankle, knee, and hip similar to Gribble et al. ${ }^{8} \mathrm{He}$ noted that fatigued individuals are at greater risk for injury because of loss of balance, and suggested that avoidance of fatigue and preconditioning may help limit injury. Myers et al. ${ }^{51}$ used isokinetic fatigue of the shoulder rotators to conclude that fatigue of the internal and external rotators of the shoulder led to decreased proprioception of the shoulder but had no significant effect on neuromuscular control. Gribble and Hertel ${ }^{7}$ used isokinetic fatigue of the saggital plane movers of the ankle, knee and hip to show that localized fatigue to these areas has an effect on center of pressure velocity during static postural control using a single leg stance. 
Table B1. Isokinetic Fatiguing Protocols 7,8,21,22,51,65

\begin{tabular}{|c|c|c|c|c|c|}
\hline Authors & $\begin{array}{c}\text { Purpose of } \\
\text { Study }\end{array}$ & Subjects & Fatigue Protocol & Results & Conclusions \\
\hline Gribble $^{8}$ & $\begin{array}{l}\text { Investigate the } \\
\text { effects of } \\
\text { fatigue and } \\
\text { CAI on } \\
\text { performance } \\
\text { measures of a } \\
\text { dynamic } \\
\text { postural } \\
\text { control task }\end{array}$ & $\begin{array}{l}30 \\
\text { subjects } \\
(16 \\
\text { healthy, } \\
14 \text { CAI) }\end{array}$ & $\begin{array}{l}\text { Concentric- } \\
\text { concentric } \\
\text { PF/DF, knee } \\
\text { FLEX/EXT, hip } \\
\text { FLEX/EXT. } \\
\text { Movement } \\
\text { pattern at } \\
60^{\circ} / \text { second until } \\
\text { below } 50 \% \text { of } \\
\text { peak torque }\end{array}$ & $\begin{array}{l}\text { Involved side of } \\
\text { CAI displayed } \\
\text { significantly } \\
\text { smaller reach } \\
\text { values and knee } \\
\text { FLEX angles for } \\
\text { all reach groups } \\
\text { compared to the } \\
\text { uninjured side }\end{array}$ & $\begin{array}{l}\text { CAI and fatigue } \\
\text { disrupted } \\
\text { dynamic postural } \\
\text { control, most by } \\
\text { altering control } \\
\text { of the saggital- } \\
\text { plane joint } \\
\text { angles proximal } \\
\text { to the ankle }\end{array}$ \\
\hline $\begin{array}{l}\text { Yaggie and } \\
\text { McGregor }\end{array}$ & $\begin{array}{l}\text { To quantify } \\
\text { changes in } \\
\text { balance } \\
\text { parameters and } \\
\text { ranges of } \\
\text { postural } \\
\text { control at the } \\
\text { ankle after } \\
\text { isokinetic } \\
\text { fatigue }\end{array}$ & $\begin{array}{l}24 \text { men } \\
\text { without } \\
\text { ankle } \\
\text { trauma } \\
\text { within } 2 \\
\text { years }\end{array}$ & $\begin{array}{l}\text { Fatigue of the } \\
\mathrm{PF} / \mathrm{DF} \text { in the } \\
\text { eccentric- } \\
\text { concentric mode } \\
\text { at } 60^{\circ} / \text { second } \\
\text { until below } 50 \% \\
\text { of maximum } \\
\text { joint torque }\end{array}$ & $\begin{array}{l}\text { Mediolateral sway } \\
\text { greatest at time } 0, \\
\text { total sway } \\
\text { increased at all } \\
\text { time points } \\
\text { postfatigue. } \\
\text { For lean test } \\
\text { fore/aft sway } \\
\text { increased at time } 0 \\
\text { and time } 10 \text { and } \\
\text { total sway } \\
\text { increased at all } \\
\text { time points }\end{array}$ & $\begin{array}{l}\text { Isokinetic fatigue } \\
\text { of the ankle } \\
\text { PF/DF } \\
\text { significantly } \\
\text { influences sway } \\
\text { parameters and } \\
\text { ranges of } \\
\text { postural control } \\
\text { in healthy men }\end{array}$ \\
\hline $\begin{array}{l}\text { Johnston et } \\
\text { al. }{ }^{65}\end{array}$ & $\begin{array}{l}\text { To determine } \\
\text { whether lower } \\
\text { extremity } \\
\text { fatigue affects } \\
\text { the ability to } \\
\text { balance on an } \\
\text { unstable } \\
\text { platform }\end{array}$ & $\begin{array}{l}20 \text { healthy } \\
\text { subjects }\end{array}$ & $\begin{array}{l}\text { Fatigued to less } \\
\text { than } 50 \% \text { of their } \\
\text { maximal tested } \\
\text { strength on an } \\
\text { isokinetic } \\
\text { dynamometer at } \\
\text { 1-min intervals } \\
\text { of various speeds }\end{array}$ & $\begin{array}{l}\text { Significant } \\
\text { decreases in motor } \\
\text { control } \\
\text { performance on the } \\
\text { three static tests } \\
\text { following exercise } \\
\text { to fatigue in all } \\
\text { subjects }(\mathrm{P}<0.001)\end{array}$ & $\begin{array}{l}\text { Fatigued } \\
\text { individuals are at } \\
\text { an increased risk } \\
\text { of injury because } \\
\text { of loss of } \\
\text { balance. }\end{array}$ \\
\hline$\underset{51}{\text { Myers et al. }}$ & $\begin{array}{l}\text { To examine } \\
\text { effects of } \\
\text { fatigue on } \\
\text { proprioception } \\
\text { and } \\
\text { neuromuscular } \\
\text { control of the } \\
\text { shoulder }\end{array}$ & $\begin{array}{l}32 \text { college } \\
\text { age } \\
\text { subjects } \\
\text { (16 male, } \\
16 \text { female) }\end{array}$ & $\begin{array}{l}\text { Continuous } \\
\text { concentric IR } \\
\text { and ER exercises } \\
\text { at } 180^{\circ} / \text { second } \\
\text { until } 3 \\
\text { consecutive reps } \\
\text { below } 50 \% \text { of } \\
\text { maximum peak } \\
\text { torque }\end{array}$ & $\begin{array}{l}\text { Significant } \\
\text { difference for } \\
\text { absolute angular } \\
\text { error, no significant } \\
\text { difference for sway } \\
\text { velocity }\end{array}$ & $\begin{array}{l}\text { Fatigue of the IR } \\
\text { and ER } \\
\text { decreases } \\
\text { proprioception in } \\
\text { the shoulder but } \\
\text { has no effect on } \\
\text { neuromuscular } \\
\text { control }\end{array}$ \\
\hline $\begin{array}{l}\text { Gribble and } \\
\text { Hertel }^{7}\end{array}$ & $\begin{array}{l}\text { To examine } \\
\text { the effects of } \\
\text { fatigue on } \\
\text { lower } \\
\text { extremity } \\
\text { postural } \\
\text { control during } \\
\text { single leg } \\
\text { stance }\end{array}$ & $\begin{array}{l}14 \text { healthy } \\
\text { subjects }\end{array}$ & $\begin{array}{l}\text { Concentric- } \\
\text { concentric } \\
\text { PF/DF, knee } \\
\text { FLEX/EXT, hip } \\
\text { FLEX/ EXT } \\
\text { Movement } \\
\text { pattern at } \\
60^{\circ} / \text { second until } \\
\text { below } 50 \% \text { of } \\
\text { peak torque }\end{array}$ & $\begin{array}{l}\text { Fatigue at the knee } \\
\text { and hip led to } \\
\text { postural control } \\
\text { impairment in the } \\
\text { frontal plane, } \\
\text { fatigue at all three } \\
\text { joints led to } \\
\text { postural control } \\
\text { impairment in the } \\
\text { saggital plane }\end{array}$ & $\begin{array}{l}\text { There is an effect } \\
\text { of localized } \\
\text { fatigue on the } \\
\text { sagittal plane } \\
\text { movers of the } \\
\text { lower extremity } \\
\text { on center of } \\
\text { pressure } \\
\text { excursion } \\
\text { velocity. }\end{array}$ \\
\hline
\end{tabular}




\begin{tabular}{|c|c|c|c|c|c|}
\hline $\begin{array}{l}\text { Wikstrom } \\
\text { et al. }{ }^{24}\end{array}$ & $\begin{array}{l}\text { To compare } \\
\text { effects of } \\
\text { isokinetic } \\
\text { fatigue and } \\
\text { functional } \\
\text { fatigue on time } \\
\text { to stabilization } \\
\text { (TTS) ground } \\
\text { reaction force } \\
\text { (GRF), and } \\
\text { joint } \\
\text { kinematics } \\
\text { during a jump } \\
\text { landing }\end{array}$ & $\begin{array}{l}20 \text { healthy } \\
\text { subjects ( } 8 \\
\text { male, } 12 \\
\text { female) }\end{array}$ & $\begin{array}{l}\text { The isokinetic } \\
\text { portion of the } \\
\text { fatigue was } \\
\text { performed using } \\
\text { concentric- } \\
\text { concentric } \\
\text { contractions of } \\
\text { the PF and DF as } \\
30 \% / \text { sec, and } \\
120^{\circ} / \text { sec } \\
\text { respectively until } \\
3 \text { consecutive } \\
\text { contractions } \\
\text { below } 50 \% \\
\text { pretest values }\end{array}$ & $\begin{array}{l}\text { No significant } \\
\text { difference when } \\
\text { comparing TTS, } \\
\text { GRF, and joint } \\
\text { kinematics after } \\
\text { isokinetic and } \\
\text { functional fatigue } \\
\text { protocols }\end{array}$ & $\begin{array}{l}\text { No difference } \\
\text { between } \\
\text { isokinetic and } \\
\text { functional } \\
\text { fatigue protocols } \\
\text { relative to } \\
\text { dynamic stability } \\
\text { when landing } \\
\text { from a jump }\end{array}$ \\
\hline
\end{tabular}

Legend: PF: Plantarflexion; DF: Dorsiflexion; FLEX: Flexion; EXT: Extension; IR: Internal Rotation; ER: External Rotation

Some authors may decide to use a repetitive task or repeated movement fatiguing protocol instead of isokinetic fatigue. One reason a clinician may use this is if they do not have access to a dynamometer to complete an isokinetic fatiguing protocol. Sparto et al. ${ }^{66}$ looked at a repetitive lifting task. They used a LIDOLift lifting simulator to determine maximal isoinertial capacity. Then the subjects lifted a small box with handles centered on the side that was attached to a robotic arm. The simulator was set to provide $25 \%$ of the subject's maximal isoinertial lifting capacity. The subjects then were instructed to lift the box at his maximal rate and continue until 1) they could no longer continue, or 2) their heart rate reached 180 beats/minute as measured by the examiner. They found that fatigue led to a decreased range of motion at the knee and hip combined with increased peak lumbar flexion which indicated the change from a squat lift to a stoop lift. They went further to suggest that fatigue could lead to back injury due to improper lifting technique. Corbeil et al. ${ }^{25}$ also used a repeated lifting fatiguing protocol in their research. They were looking at the perturbation of the postural control system induced by muscular fatigue. Their fatigue protocol consisted of fatiguing the ankle plantarflexors with repeated 
plantarflexion of both legs. The subjects sat at a standard ankle training device and had to lift a bar upward by lifting their heels off of the ground. The subjects had to perform 100 repetitions starting at $75 \%$ of their maximal workload using a reverse pyramidal technique in which the load is diminished gradually when the subjects can no longer perform plantarflexion. They found that this fatigue to the ankle plantarflexors increased postural sway (faster center of pressure velocity and greater center of pressure mean and median frequencies).

A more popular and easier technique to fatigue a subject is through an isometric contraction. This method is best for a clinician who does not have the financial resources to purchase equipment and can be easily performed at a college or university. Isometric contractions are defined as a contraction of a single muscle or group of muscles against resistance that produces no change in muscle length. The most popular way to fatigue the calf muscles using this technique is to have the subjects stand on their tiptoes until they can no longer keep their heels off the ground. Four such studies used this exact method of fatigue, where all of the authors but one indicated a negative effect of fatigue. The first study by Vuillerme et al. ${ }^{20}$ looked at attentional demands and postural sway and their affects on calf muscle fatigue. For their research they used nine healthy university age subjects. Their testing method was to have them respond to an unpredictable auditory stimulus while maintaining stable postures. Their results showed that after a fatiguing protocol, there was a significant shift of mean position of center of pressure. From these results they were able to conclude that muscular fatigue may put individuals at high risk of falling especially when engaging in concurrent tasks. 
In another study by Vuillerme et al. ${ }^{26}$ postural sway under muscle vibration and muscle fatigue in humans was evaluated. They hypothesized that muscle vibration under fatigue should lead to an even larger increase in postural sway. For their research they used ten healthy university age students. Using a force plate they had their participants stand upright with their eyes closed in a relaxed position. Once they assumed the position they turned on vibrators for 10 seconds and placed them on the tendons of gastrocnemius, soleus, and tibialis anterior. Their results indicated a similar increase in postural sway under muscle fatigue as well as under muscle vibration. To account for the rejection of the hypothesis, they postulated that fatigued muscles are less sensitive to muscle vibration and the central nervous system relies less upon proprioceptive information originating from fatigued muscles for regulating posture. ${ }^{26}$

Patikas et al. ${ }^{27}$ evaluated electromyographic changes of agonist and antagonist calf muscles during maximum isometric induced fatigue. They used nine healthy subjects that were completely untrained to the task. They recorded EMG activity of the soleus and anterior tibialis during a maximal isometric heel lift fatiguing protocol. They noted that the soleus EMG activity decreased during the task, and the anterior tibialis EMG activity indicated a small, but non-significant decrease. However, the ratio of the anterior tibialis to the soleus EMG increased significantly at the end of the protocol. From these results the authors were able to state that during a maximal fatiguing protocol, both the agonist and antagonist muscle activity declines, however, the slower rate of antagonist decline needs to be examined. Further, they were able to suggest that this may reflect a higher level of agonist and antagonist co-activation and probably a relatively opposing torque from the antagonist muscles at the end of the session. ${ }^{27}$ 
Huston et al. ${ }^{19}$ evaluated the effects of calf muscle fatigue on saggital plane joint position sense in the ankle. In his study the authors used a $2 \times 2$ factorial design with 20 healthy subjects. The subjects testing session consisted of trials at $10^{\circ}$ of dorsiflexion, and $20^{\circ}$ of plantarflexion both before and after the fatiguing protocol. These authors noted that there were no significant main effect for the condition, measurement, or interaction of the two. From this they were able to hypothesize that since there is no significant difference between conditions, the anterior tibialis may be the main controller of joint position sense. 19

Other methods of isometric fatigue performed by Forestier et al. ${ }^{22}$ and Kawakami et al. ${ }^{28}$ also indicate similar detrimental results. Forestier et al. ${ }^{22}$ examined alteration of the position sense at the ankle when induced by muscular fatigue. In their study, they fatigued the anterior tibialis muscle by having the subjects maintain an isometric contraction of the anterior tibialis, until they could not maintain $70 \%$ on the strain gauge for 15 seconds. Their results indicated that joint position sense at the ankle is reduced following muscular fatigue of the anterior tibialis. Another study conducted by Kawakami et al. ${ }^{26}$ evaluated fatigue responses of human triceps surae muscles during repetitive maximal isometric contractions. ${ }^{28}$ They had their subjects perform 100 consecutive isometric contractions of the plantarflexors in both knee positions of $0^{\circ}$ of flexion, and $90^{\circ}$ of flexion. ${ }^{63}$ They noted that fatigue lead to a decrease in plantar flexion torque due to both general and peripheral fatigue and also went on to note that greater fatigability in $0^{\circ}$ of knee flexion could result from a greater contribution of the gastrocnemius, and therefore more pronounced fatigue of this muscle. 
The last technique to fatigue the body is functional fatigue. Functional fatigue is difficult to evaluate because there is no assurance that the part of the body being tested is actually fatigued. Lepers et al. ${ }^{29}$ looked at neuromuscular fatigue during a long-duration cycling exercise. They used endurance-trained cyclists and had them cycle for five hours at $55 \%$ of their maximum aerobic power. Through their research they noted that contractile properties are significantly altered after the first hour, and excitability and central drive are more impaired toward the latter stages of exercise.

The fatigue protocol that appears to be more functional by fatiguing all energy systems has been used by Susco et al. ${ }^{14}$ and Wilkins et al. ${ }^{15}$ This fatiguing protocol is more representative of what athletes do on a daily basis. Susco et al. ${ }^{14}$ looked at balance after exertion as measured by the Balance Error Scoring System (BESS), while Wilkins et al. ${ }^{15}$ looked at performance on the BESS and how it decreases after fatigue. Both studies used a seven station fatigue protocol to simulate exercise during the activity. Station one consisted of a five-minute moderate jog at the subjects self-selected pace. Station two was three minutes of sprints up and down the length of a basketball court. Station three was two minutes of push-ups. Stations four was two minutes of sit-ups. Station five was three minutes of 12 inch step-ups. Stations six was another three minutes of sprints, and station seven was a two minute run at as fast a pace as they could maintain for the entire two minutes. To ensure that adequate fatigue was attained, the clinicians used the 15-point Borg scale to measure each subjects Rating of Perceived Exertion. They took a measure before station one, after station 3 at the mid-point, and directly after the last station. Adequate fatigue was deemed to be an RPE of 15 and they found that this represents $75 \%$ to $90 \%$ maximum oxygen consumption. 
Wilkins et al. ${ }^{15}$ noted that BESS scores increased immediately after the fatigue protocol demonstrating that balance was indeed affected by fatigue and therefore the BESS should not be administered as a part of a concussion evaluation immediately after exercise. ${ }^{15}$ However Susco et al. ${ }^{14}$ found that BESS performance in healthy individuals will return to baseline levels within 20 minutes following exertion. As demonstrated by most of the studies described, fatigue does in fact have an impact on postural control. How large of an impact it has is a question that is always under investigation. The studies described also presented that fatigue can have an impact on static postural control, semi-dynamic postural control, as well as dynamic postural control. The effects of local or peripheral fatigue in that fatigued local muscles such as the ankle plantarflexors when standing on the tip toes 19,22,26,27 is not clear exactly how local muscular fatigue affects postural control. As defined above, fatigue is the reduction in the force-generating capacity regardless of the task performed. ${ }^{25,32}$ "At the peripheral level the pre- and postsynaptic mechanism and sites are potentially implicated including a failure in the transmission of the neural signal or a failure of the muscle to respond to neural excitation." ${ }^{25}$ At the central level, fatigue may also cause a failure of the excitation of motoneurons due to changes in the nervous system. 25

The contribution of joint and muscle mechanoreceptors also is controversial. It seems that the greatest contribution to proprioception is from the muscle receptors, specifically the muscle spindles, and golgi tendon organs. Since fatigue protocols affect muscle tissue more than articular receptors, diminished proprioception, and therefore postural control may conceptually be thought of as secondary to loss of muscle receptor input. ${ }^{33} \mathrm{It}$ is also theorized that muscle fatigue may impair the proprioceptive and 
kinesthetic properties of joints by, "increasing the threshold of muscle spindle discharge, disrupting afferent feedback, and subsequently altering conscious joint awareness." 7

The information presented in the section about functional fatigue indicated that general fatigue also plays a role in altering postural control. Although the effects of functional fatigue are much more difficult to quantify, the authors represented have been able to show that long bouts of cycling induce neuromuscular fatigue, and that 30 minutes of station exercise can alter balance performances on the BESS. ${ }^{14,15,29}$

Star Excursion Balance Test

The Star Excursion Balance Test has been shown to be sensitive for screening musculoskeletal injury such as chronic ankle instability, strength deficits of the quadriceps, and patellofemoral pain syndrome. ${ }^{2,11}$ Earl and Hertel ${ }^{11}$ demonstrated that with the exception of the gastrocnemius, muscle activation during the SEBT is direction dependent.

${ }^{11}$ As with any rehabilitation, the clinician wants to re-establish neuromuscular control following an injury. Most clinicians support the fact that the best way to do this is through closed kinetic chain (CKC) exercises.

The SEBT allows cocontraction of the hamstring and quadriceps during all directions of the test. ${ }^{11}$ The quadriceps are most active during the three anterior excursions. This is because in order to perform the anterior excursions, the subjects must lean back, extending the trunk in order to maintain their balance. At this point gravity will act on the upper body causing a, "large knee-flexion moment, which must be controlled by an extension moment produced by the quadriceps." ${ }^{11}$ More specifically, vastus lateralis activity is highest during 
Figure B1. Star Excursion Balance Test ${ }^{2}$

Left-Leg Stance

A

$\mathrm{AL}$

$\mathrm{L}$

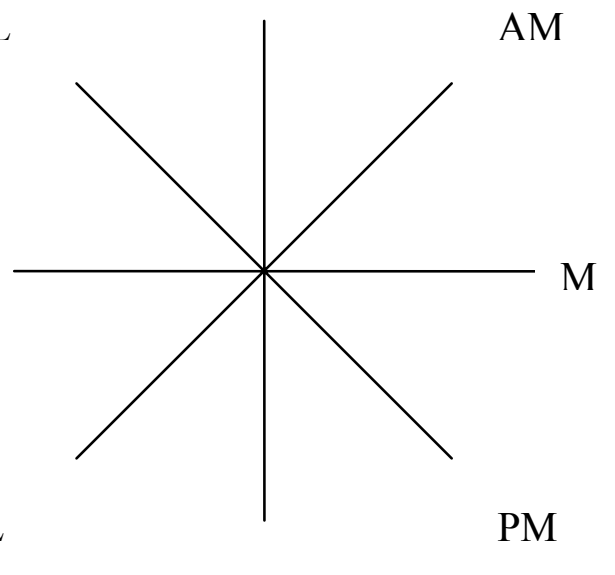

Right-Leg Stance

A

$\mathrm{AM}$

$\mathrm{M}$

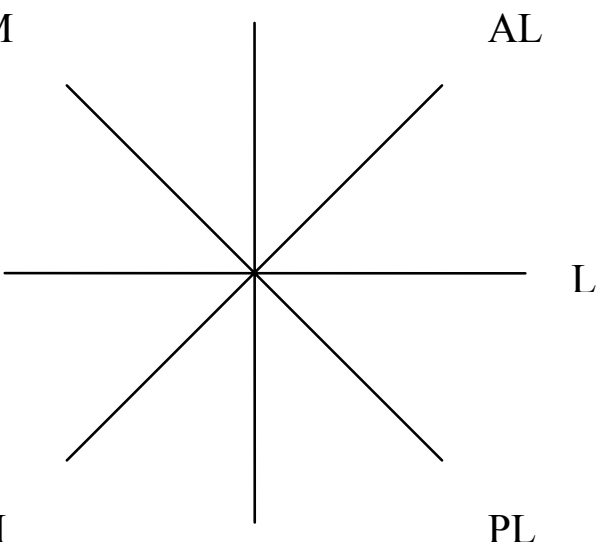

$\mathrm{P}$

Legend: A: anterior excursion; AL: anterolateral excursion; L: lateral excursion; PL: posterolateral excursion; P: posterior excursion; PM: posteromedial excursion; M: medial excursion; AM: anteromedial excursion.

the medial and posteromedial excursions. This is most likely the result of muscular stabilization that is performed against the varus force that is created by performing these excursions. With this information in mind, it is apparent that performing the anteriorly and medially directed excursions would be a way to eccentrically strengthen the quadriceps and thus improve dynamic control in the later stages of injury rehabilitation. Another common ailment of the lower extremity is patellofemoral pain syndrome. One of the major goals of rehabilitation for this injury is to strengthen the vastus medalis oblique (VMO). When performing the SEBT, the VMO is highly active during all of the anteriorly directed excursions.

The biceps femoris is also active during the SEBT. It is most active during the posterolateral, posterior, and lateral excursions. ${ }^{11}$ This can be explained by the effects of 
gravity acting on the trunk causing a hip-flexion moment. To perform the posterior excursions the athlete must flex his or her trunk in order to extend the leg backward. To do this the hamstrings must eccentrically contract as a way to resist the hip-flexion moment. The lateral excursion involves extreme external rotation of the hip to perform, thus also leading to high levels of activity in the biceps femoris.

The SEBT can also be used in ACL rehabilitation. During rehabilitation of an ACL, the amount of strain that is placed on the ACL is very little and must be closely monitored. Performing open kinetic chain (OKC) exercises creates a lot of shear force on the new ACL and can be dangerous to perform. ${ }^{11}$ This is the reason why most rehabilitation exercises are performed in the CKC. This helps to lower the anterior shear forces at the tibiofemoral joint. During the SEBT, the anterior tibialis is most active during the three posteriorly directed excursions. In order to perform these excursions, the center of mass must shift posteriorly on the foot, so the anterior tibialis keeps the knee and leg over the center of pressure to prevent a posteriorly directed fall. ${ }^{11}$

Since balance is an important component of the athlete's everyday activity, having a way to measure this is important. Measurements of static postural control are useful but play a limited role for athlete's who perform their skills in a highly dynamic setting. The SEBT can be a useful tool in measurement of dynamic postural control as it has high intrarater reliability, but it can also be a useful tool in the rehabilitation of athletic injuries. Summary

Proprioception is the specialized variation of the sensory modality of touch that encompasses the sensation of joint movement. The application of proprioception requires afferent information from the many mechanoreceptors like the muscle spindles, golgi 
tendon organs, Ruffini endings, Pacinian Corpuscles, and cutaneous receptors.

Proprioception requires the peripheral nervous system to house the mechanoreceptors and send signals to the spinal cord. The central nervous system, which consists of the brain and spinal cord, sends signals from the motor cortex, down the descending tracts and out to the muscles via motor neurons. Neuromuscular control is the efferent response to the afferent stimuli presented by the mechanoreceptors. It is represented by the muscle movement in response to the muscle stretch signaled by the muscle spindle.

Postural control is the ability to maintain a stable base of support and can either be static (maintaining a stable base of support while not moving) or dynamic (maintaining a stable base of support while completing a functional task). The only known test of dynamic postural control is the Star Excursion Balance Test. This test requires subjects to maintain a stable base of support while reaching out with the opposite foot in eight predetermined directions. We know that the SEBT is a reliable test, and can also provide input on injury status and can be used in rehabilitations.

Fatigue is a reduction in the force-generating capacity regardless of the task performed. Fatigue can be either central or peripheral in nature, with peripheral fatigue being the more likely cause of fatigue in athletes. There are a plethora of different ways to fatigue the body whether it is through isokinetic contractions, isometric contractions, repeated contractions, or through functional means. Fatigue is a part of athletics and does indeed play a role in decreasing postural control which can be measured by the SEBT. 
APPENDIX C

ADDITIONAL METHODS

Table C1. Informed Consent

CONSENT AND INFORMATION FORM

THE EFFECTS OF ACTIVITY RELATED FATIGUE ON DYNAMIC POSTURAL COTROL AS MEASURED BY THE STAR EXCURSION BALANCE TEST

\section{Introduction}

I, , have been invited to participate in this research study, which has been explained to me by Timothy A. Kulpa, BS, ATC. He is conducting this research under the supervision of Michelle A. Sandrey, $\mathrm{PhD}$, ATC to fulfill the requirements for a master's thesis in Athletic Training in the School of Physical Education at West Virginia University.

\section{Purpose of the Study}

The purpose of this study is to examine how dynamic postural control is affected in a nonfatigued and fatigued condition as measured by the Star Excursion Balance Test for the lower extremity.

\section{Description of Procedures}

This study will be conducted in the Athletic Training Research Laboratory, and on the main Coliseum basketball floor at West Virginia University, Morgantown, WV 26506.

Orientation Procedures

At an orientation meeting, I will be explained the purpose of this study. I will also be given a demographic/injury history questionnaire and an informed consent form explaining my rights as a research subject.

If I am an eligible subject, I will be contacted by the principle investigator and asked to attend a second meeting. At the second meeting I will be given an opportunity to familiarize myself with the testing procedures. The testing procedure is the Star Excursion Balance Test, which is a test that measures dynamic postural control. The fatiguing protocol is functional in nature and will consist of seven stations spanning a total time of 20 minutes.

$\underline{9 / 24 / 05}$

Page 1 of 5

Version Date

$\overline{\text { Initials }} \quad \overline{\text { Date }}$




\section{THE EFFECTS OF ACTIVITY RELATED FATIGUE ON DYNAMIC POSTURAL COTROL AS MEASURED BY THE STAR EXCURSION BALANCE TEST}

After the familiarization period I will have my leg length measured from my front of my hip bone to bone on the inside of my ankle so that my reach measures can be normalized and therefore generalized to the population. I will then undergo the Star Excursion Balance Test on my dominant leg and the measures will be recorded. My dominant foot will be identified as the leg I would use to kick a soccer ball. During the test I will be standing in the middle of a grid with my dominant leg barefoot.

While standing on my dominant foot, I will complete six practice trials in each of the eight directions (Anterior, Anterolateral, Lateral, Posterolateral, Posterior, Posteromedial, Medial, and Anteromedial). After this is completed the examiner will pick a starting point at random out of a hat. I will perform three trials in each direction traveling in a clockwise direction if my left foot is my dominant leg, and in the counterclockwise direction if my right leg is my dominant leg from the randomized starting point, and the measures will be averaged to obtain my score for each trial. To complete each trail, I will balance on my dominant leg and reach my non-dominant leg in the prescribed direction. I will touch my foot down at the furthest point I can reach in a controlled manner, and then come back to the starting position without compromising my base of support. I understand that if I loose my balance, touch down in an uncontrolled manner, or fail to come back to the starting position that this trial will be thrown out, and I will have to complete another trial in that direction.

After completing the Star Excursion Balance Test I will complete a five minute warm-up on a bike, cycling at level two. After this warm-up period I will complete a stretch band routine that consists of stretching my hamstrings, quadriceps, hip flexors, groin, gluteals, and calf muscles. Next I will identify my rating of perceived exertion (RPE) on a chart, and my response will be recorded. I will then undergo a fatiguing procedure that is functional in nature and will last for a total of 20 minutes. The protocol for the fatiguing procedure will consist of seven stations. The first station will consist of jogging at a self-selected pace for five minutes, station two consists of sprinting up an down a basketball court for three minutes, station three is two minutes of push-ups. After station three, I will again identify my RPE, and it will be recorded. I will then proceed with the remaining ten minutes of the fatiguing protocol. Station four will consist of two minutes of sit-ups. Station five will be three minutes of 12 inch step-ups. Station six will consist of three more minutes of sprints. Finally, station seven will consist of two minutes of running at as fast a pace as I can maintain for the entire two minutes. As soon as I finish the seventh station, I will again identify my RPE, and it will be recorded as I walk to the post testing area.

$\underline{9 / 24 / 05}$

Page 2 of 5

Version Date

$\overline{\text { Initials }} \overline{\text { Date }}$ 


\section{THE EFFECTS OF ACTIVITY RELATED FATIGUE ON DYNAMIC POSTURAL COTROL AS MEASURED BY THE STAR EXCURSION BALANCE TEST}

I will then undergo the same testing procedure as before using the Star Excursion Balance Test. However this time I will not perform the practice trials. I understand that I will again be asked to stand in the middle of the grid on my dominant foot. The examiner will again pick a starting point at random using a random number table.

I will perform three trials in each direction traveling in a clockwise or counterclockwise direction based on which is my dominant leg from the randomized starting point, and the measures will be averaged to obtain my score for each trial. To complete each trail, I will balance on my dominant leg and reach my non-dominant leg in the prescribed direction. I will touch my foot down at the furthest point I can reach in a controlled manner, and then come back to the starting position without compromising my base of support. I understand that if I loose my balance, touch down in an uncontrolled manner, or fail to come back to the starting position that this trial will be thrown out, and I will have to complete another trial in that direction. Once testing is complete I will be allowed to leave. I understand that West Virginia University researchers hope to enroll approximately 30 people in this study. I further understand that the approximate time to complete this study will be one hour.

\section{Risk and Discomforts}

As with any task involving fatigue and the Star Excursion Balance Test (SEBT), I understand there are minimal risks of lower leg soreness and the possibility of losing my balance from participating in this study. While performing the SEBT, it is likely that I will not lose my balance I will be performing the test with my eyes open and the principle examiner will be standing next to me.

I understand that I will warm-up and perform a stretch band routine prior to testing. This routine will isolate the hamstrings, IT band, groin, gluteals, quadriceps, hip flexors, and calf muscles. If at any time during this study I become exhausted to the point that I cannot go one any longer I will be free to end the testing session. I also understand that if at any time during this study I become light headed or dizzy that I will be free to end the testing session. I also understand that if I obtain a Rating of Perceived Exertion (RPE) of 19-20 that the principle investigator will stop the session.

I understand that every precaution has been taken to prevent me from being injured during this study. Should any injury occur I understand that Timothy A. Kulpa, BS, ATC will provide first aid and will make any necessary medical referrals at my own expense.

$\underline{9 / 24 / 05}$

Page 3 of 5

Version Date

$\overline{\text { Initials }} \quad \overline{\text { Date }}$ 


\section{THE EFFECTS OF ACTIVITY RELATED FATIGUE ON DYNAMIC POSTURAL COTROL AS MEASURED BY THE STAR EXCURSION BALANCE TEST}

\section{Alternative}

I understand that I do not have to participate in this study.

\section{Benefits}

I understand that this study is not expected to be of direct benefit to me, but the knowledge gained may be of benefit to others.

\section{Contact Persons}

For more information about this research, I can contact Timothy A. Kulpa, BS, ATC at (518) 330-8546 or at tkulpa@mix.wvu.edu or his faculty advisor, Michelle A. Sandrey PhD, ATC at (304) 293-3295 Ext. 5220 or at msandrey@mail.wvu.edu. For more information regarding my rights as a research subject, I may contact the Executive Secretary of the Review Board at (304) 293-7073.

\section{Confidentiality}

I understand that any information about me obtained as a result of my participation in this research will be kept as confidential as legally possible. Identifying information on the informed consent form and demographic/injury history questionnaire will be kept confidential by assigning a code number to each informed consent form and demographic/injury history questionnaire.

I understand that my research records and test results, just like hospital records, may be subpoenaed by court order or may be inspected by the study sponsor or federal regulatory authorities (including FDA if applicable) without my additional consent. In any publications that result from this research, neither my name nor any information from which I might be identified will be published without my consent.

\section{Voluntary Participation}

Participation in this study is voluntary. I understand that I am free to withdraw my consent to participate at any time and that such refusal to participate will not affect my future care, my employee status at West Virginia University, or my class standing as an athletic training student or grades. Refusal to participate or withdrawal will involve no penalty to me. I have been given the opportunity to ask questions about the research, and I have received answers concerning areas I did not understand. In the event new information becomes available that may affect my willingness to continue to participate in this study, this information will be given to me so I may make an informed decision about my participation.

$\underline{9 / 24 / 05}$

\section{Page 4 of 5}

Version Date

$$
\overline{\text { Initials }} \quad \overline{\text { Date }}
$$


Upon signing this form, I will receive a copy.

I willingly consent to participate in this research.

Signature of Subject

Signature of Principle Investigator

$\underline{9 / 24 / 05}$

Version Date

$\overline{\text { Date }} \overline{\text { Time }}$

Date

Time

Page 5 of 5

Initials $\quad \overline{\text { Date }}$ 


\section{Authorization to Use or Disclose Protected Health Information (PHI)}

\section{West Virginia University}

I hereby voluntarily authorize the use or disclosure of my individually identifiable health information as described below.

Patient Name:

Date of Birth:

ID Number:

IRB Protocol \#: 16752

Persons/organizations providing the protected health information (e.g. hospitals):

Timothy A. Kulpa, BS, ATC

West Virginia University Athletic Training Department

Persons/organizations receiving the information (e.g. investigators, clinical coordinators, sponsor, FDA):

Timothy A. Kulpa, BS, ATC - Primary Investigator

The following information will be used:

Injury History Questionnaire

The information is being disclosed for the following purposes (Start with the Title of the study and include additional information e.g. screening and recruiting subjects; analyzing research data, or other specified purposes):

The effects of activity related fatigue on dynamic postural control as measured by the star excursion balance test - self report of injury history for inclusion in research study

I may revoke this authorization at any time by notifying the Principal Investigator in writing at:

Timothy A. Kulpa, BS, ATC

West Virginia University

PO Box 0877

Office of Intercollegiate Athletics

Morgantown, WV 26505

If I do revoke my authorization, any information previously disclosed cannot be withdrawn. Once information about me is disclosed in accordance with this authorization, the recipient may redisclose it and the information may no longer be protected by federal privacy regulations.

Page 1 of 2 


\section{Authorization to Use or Disclose Protected Health Information (Contd.)}

I may refuse to sign this authorization form. My clinical treatment may not be affected by whether or not I sign this form. I may not be allowed to participate in the research if I do not sign the form.

This authorization will expire on the date that the research study ends. (Other options for expiration include an actual date of expiration, occurrence of a particular event, or "none" if the authorization will have no expiration date.)

Expiration date:

I will be given a copy of this authorization form.

Signature of subject or subject's legal representative (Form MUST be completed before signing)

Printed name of subject's legal representative
Relationship to the subject
\begin{tabular}{|l} 
Parent \\
Medical power of attorney/representative \\
Legal guardian \\
Health care surrogate
\end{tabular}

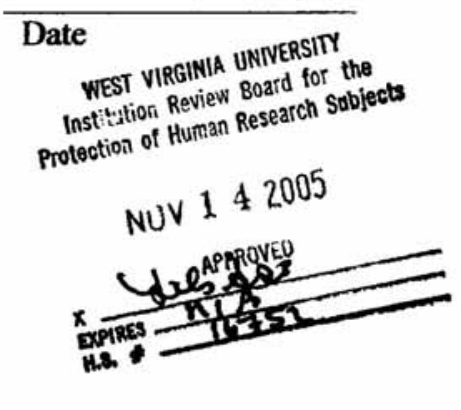

Page 2 of 2 
Table C3. Demographic/Injury History Questionnaire

\section{Demographic/ Injury History Questionnaire}

\section{Demographic}

Name:

Gender:

Age:

Height:

Weight:

Year in school: Freshman/Sophomore/Junior/Senior $/ 5^{\text {th }}$ year Senior/Graduate Student Please list all physical activities that you participate in, and how often you do them.

\section{Injury History}

1. Do you have a history of multiple ankle sprains (three or more within the last year)? YES/NO If yes, please explain:

2. Have you suffered an ankle sprain in the last 6 months that has required intervention of a medical or allied health care professional? YES/NO If yes, please explain:

3. Do you have a history of multiple knee injuries (three or more within the last year)? YES/NO If yes, please explain:

4. Have you suffered a knee injury in the last 6 months that has required intervention of a medical or allied health care professional? YES/NO If yes, please explain: 
5. Do you have a history of multiple hip injuries (three or more within the last year)? YES/NO If yes, please explain:

6. Have you suffered a hip injury in the last 6 months that has required intervention of a medical or allied health care professional? YES/NO If yes, please explain:

7. Do you have a history of head injuries (three or more in lifetime)? YES/NO If yes, please explain:

8. Have you suffered a head injury in the last 6 months that has required intervention of a medical or allied health care professional? YES/NO If yes, please explain:

9. Have you been diagnosed with any nerve disorders by a medical or allied health care professional? YES/NO If yes, please explain:

10. Have you been diagnosed with any inner ear disorders by a medical or allied health care professional? YES/NO If yes, please explain:

11. Have you been diagnosed with any visual disorders by a medical or allied health care professional? YES/NO If yes, please explain:

12. Do you currently take any medication that may affect your ability to balance? YES/NO If yes, please explain:

13. Have you been involved in any balance training programs within the last six months? YES/NO If yes, please explain: 
Table C4. Dynamic Postural Control Test using the Star Excursion Balance Test (Pre-test and Post-test) $)^{2,3,9,11}$

1. The test will begin by measuring the subjects leg length from the Anterior Superior Iliac Spine to the medial mallelous in order to normalize to leg length.

2. Subjects will be instructed to stand in the middle of the star-shaped grid and maintain their center of balance using a single-leg stance on their dominant leg, while reaching with the opposite leg as far as possible in a designated excursion.

3. Subjects will be instructed to touch the furthest point as light as possible along a chosen excursion with the most distal portion of the reach foot.

4. Subjects will be instructed to return to a bilateral stance while maintaining their balance.

5. Subjects will perform six practice trials in each of the eight possible excursions with a 10 -second rest between each excursion.

6. The subject will then rest for one minute after the practice session has been completed.

7. Three trials will be performed in each or eight excursions, with the first excursion chosen at random by choosing out of a hat and traveling in a clockwise or counter clockwise direction depending on which is their dominant leg until all eight excursions have been completed.

8. Trials will be discarded if the reach foot was used to provide considerable support when touching the ground, if the subject's stance foot is lifted from the center of the grid, or if the subject is unable to maintain their balance throughout the excursion.

9. The average score for each excursion is recorded as their reach distance. 
Table C5. Pre-Test Data Collection Sheet for the Star Excursion Balance Test

\section{Pre-Test Data Collection Sheet for the Star Excursion Balance Test}

Code:

Age:

Gender:

Height:

Weight:

Right Leg Length:

Left Leg Length:

Dominant Lower Extremity: Right/Left
Excursion
Trial 1
Trial 2
Trial 3
Average

Anterior

Anteromedial

Medial

Posteromedial

Posterior

Posterolateral

Lateral

Anterolateral

* Randomly selected first excursion 
Table C6. Fatigue Protocol ${ }^{14,15}$

1. After the pre-test SEBT, the participant will warm-up on a stationary bicycle at a selfselected pace for five minutes.

2. Participant will then perform a stretch band routine for five minutes.

3. After stretching the participant will identify his/her rating of perceived exertion (RPE)

4. Participant will begin the fatiguing protocol. Station one will consist of a five-minute moderate jog at the subjects self-selected pace. This jog will take place around the perimeter of the gym in order to help the participant maintain a steady pace.

5. Station two will be three minutes of sprints up and down the length of a basketball court.

6. Station three will be two minutes of push-ups.

7. After station three is completed, and RPE measurement will be taken since this is the half-way point.

8. Station four will consist of two minutes of sit-ups.

9. Station five will be three minutes of 12 inch step-ups.

10. Station six will consist of another three minutes of sprints up and down the basketball court.

11. Station seven will be a two minute run at as fast a pace as the participant can maintain for the entire two minutes.

12. After station seven is completed the participant will identify his/her RPE for a third time. 
Table C7. Post-Test Data Collection Sheet for the Star Excursion Balance Test

Post-Test Data Collection Sheet for the Star Excursion Balance Test

Code:

Age:

Gender:

Height:

Weight:

Right Leg Length:

Left Leg Length:

Dominant Lower Extremity: Right/Left

$\begin{array}{lllll}\text { Excursion } & \text { Trial 1 } & \text { Trial 2 } & \text { Trial 3 } & \text { Average }\end{array}$

Anterior

Anteromedial

Medial

Posteromedial

Posterior

Posterolateral

Lateral

Anterolateral

* Randomly selected first excursion 
Table C8. 15-Point Borg Scale (6-20) for measuring exertion

- $6-20 \%$ effort - Very, very light (Rest)

- $7-30 \%$ effort

- $8-40 \%$ effort

- $9-50 \%$ effort - Very light - gentle walking

- $10-55 \%$ effort

- $11-60 \%$ effort - Fairly light

- $12-65 \%$ effort

- $13-70 \%$ effort - Moderately hard - steady pace

- $14-75 \%$ effort

- $15-80 \%$ effort - Hard

- $16-85 \%$ effort

- $17-90 \%$ effort - Very hard

- $18-95 \%$ effort

- $19-100 \%$ effort - Very, very hard

- 20 - Exhaustion

Table C9. Rating of Perceived Exertion (RPE) Collection Sheet

Rating of Perceived Exertion (RPE) Collection Sheet

Code:

Age:

Gender:

Height:

Weight:

First RPE Identified - Just prior to beginning Station 1:

Second RPE Identified - After Station 3 at mid-way point:

Third RPE Identified - Immediately after Station 7: 
Figure C1. Stretch Band Routine

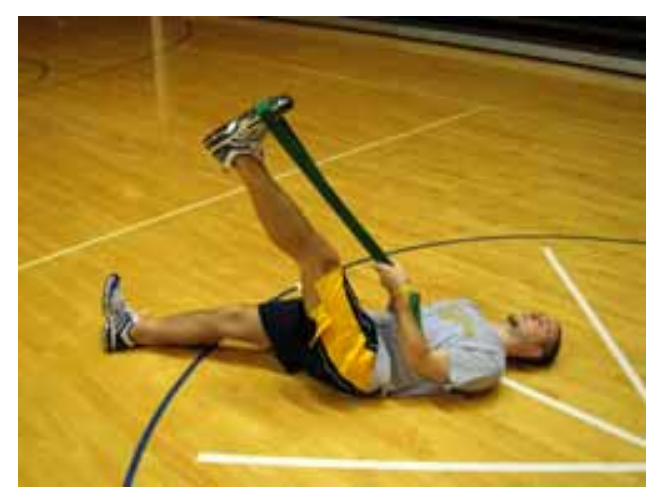

Hamstring Stretch

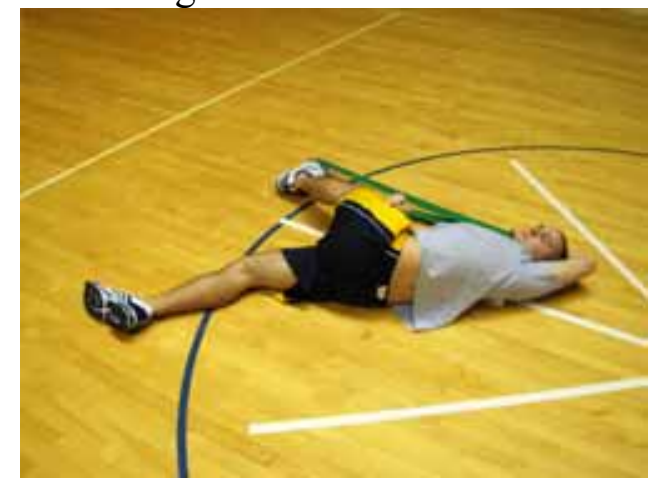

IT Band Stretch

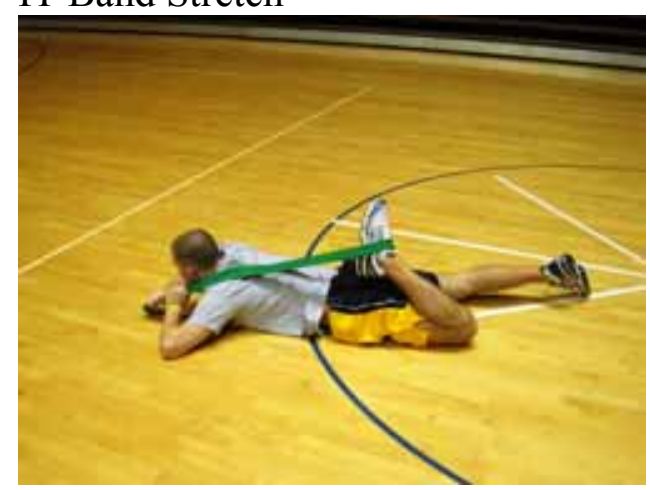

Quadricep Stretch

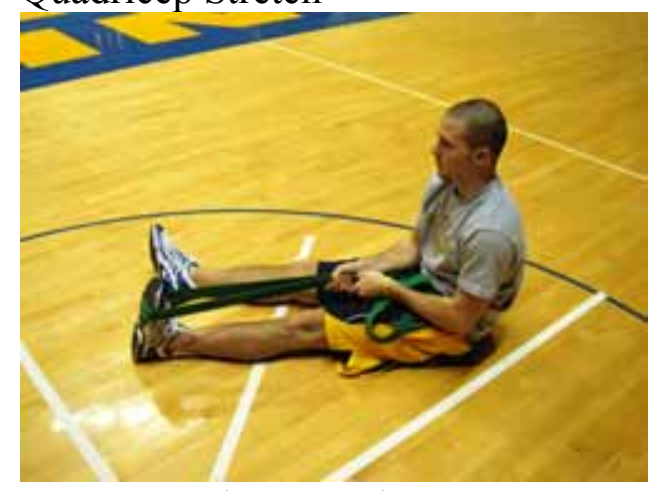

Gastrocnemius Stretch

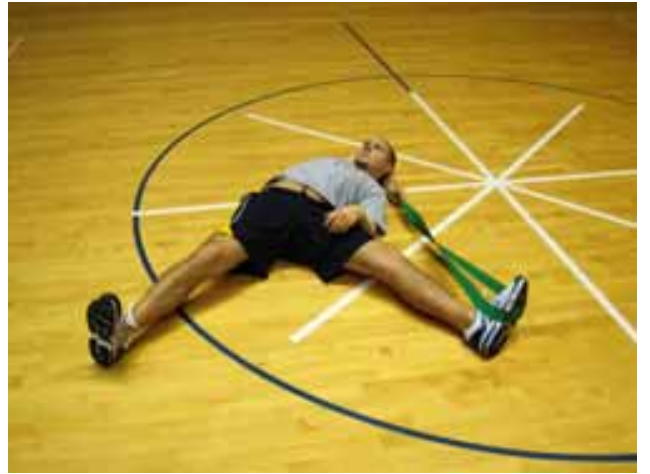

Adductor Stretch

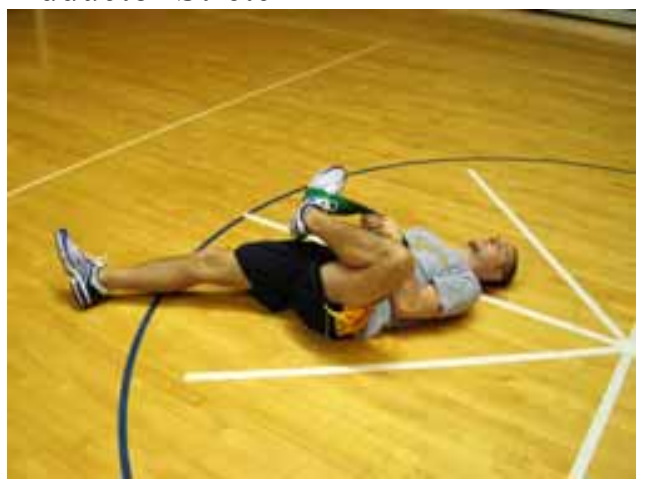

Glute Stretch

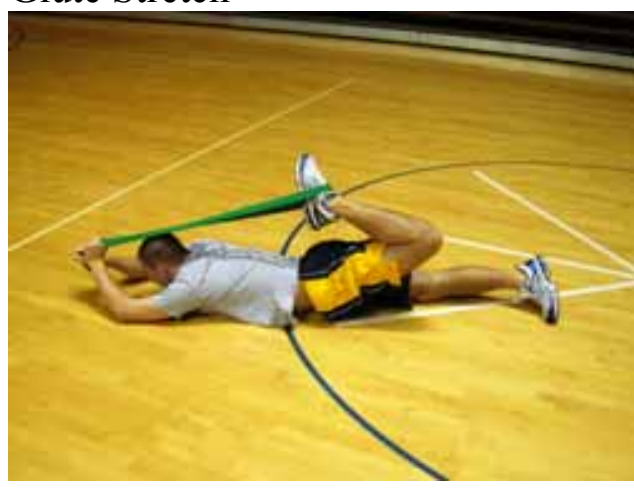

Hip Flexor Stretch

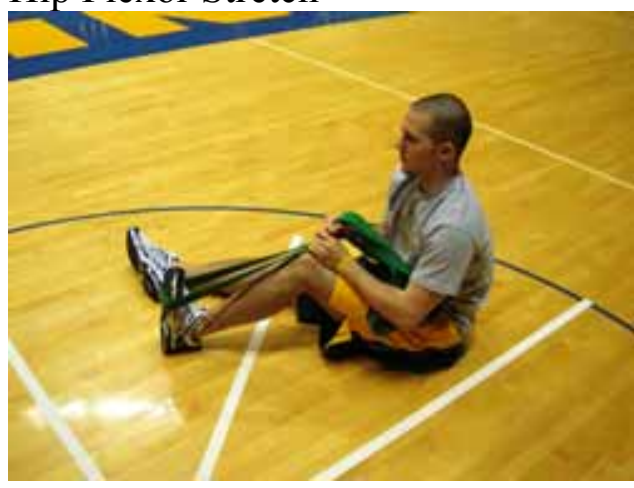

Soleus Stretch 
Figure C2. Star Excursion Balance Test ${ }^{2}$

Left-Leg Stance

A

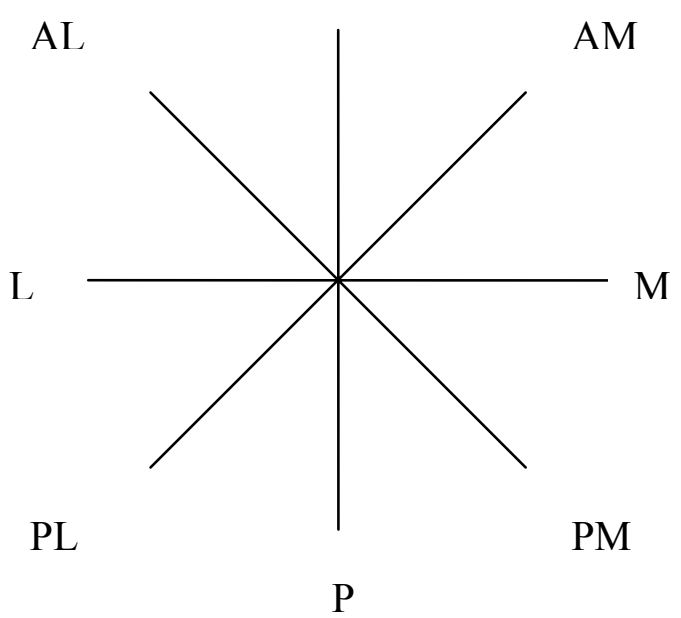

Right-Leg Stance

A

$\mathrm{AM}$<smiles>C1CCCCC1</smiles>
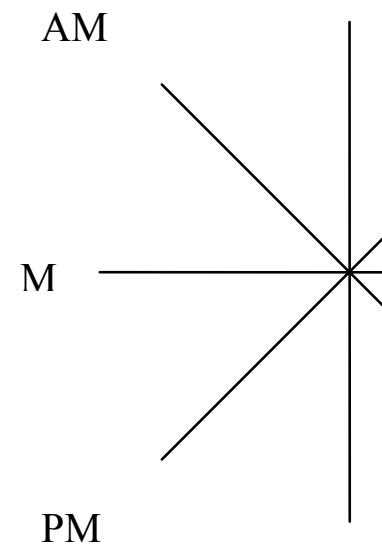

$\mathrm{P}$
$\mathrm{AL}$

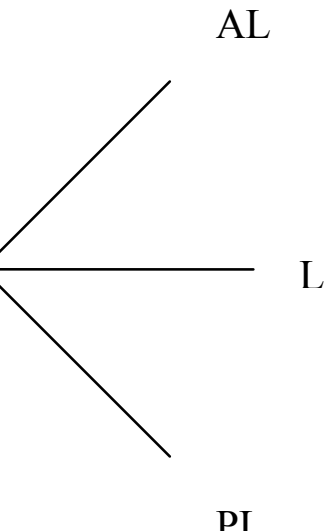

PL

Abbreviations: A: anterior excursion; AL: anterolateral excursion; L: lateral excursion; PL: posterolateral excursion; P: posterior excursion; PM: posteromedial excursion; M: medial excursion; AM: anteromedial excursion. 
Figure C3. Star Excursion Balance Test Pictures ${ }^{2}$

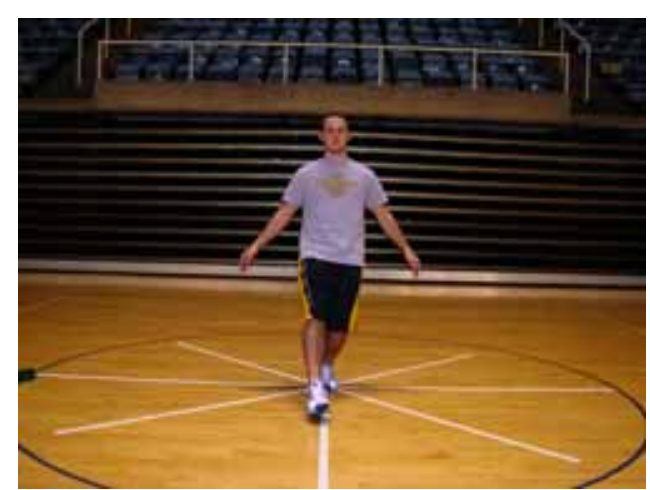

SEBT in Anterior Direction

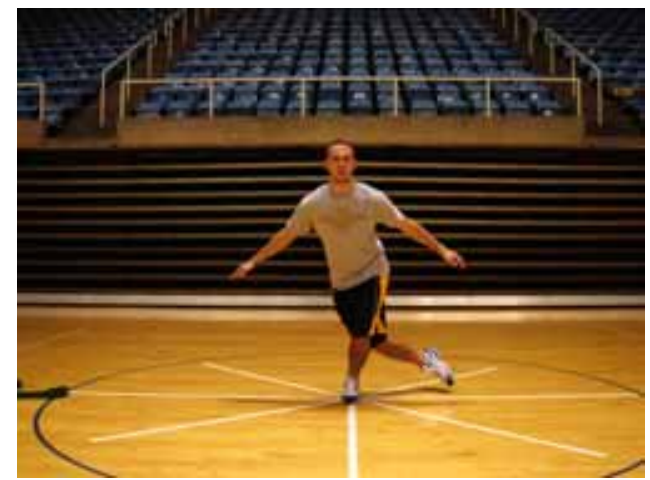

SEBT in Lateral Direction

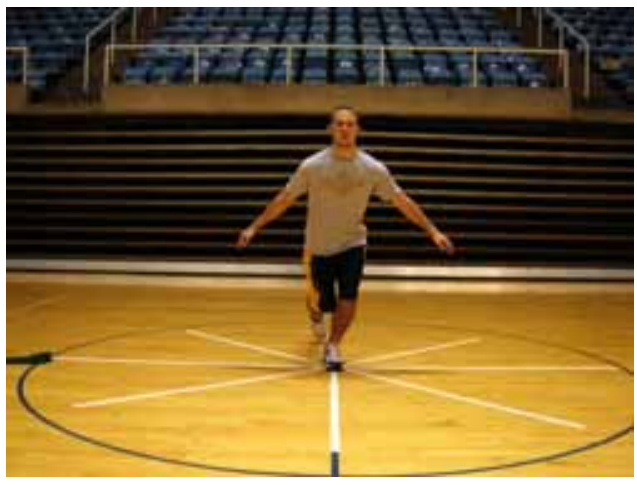

SEBT in Posterior Direction

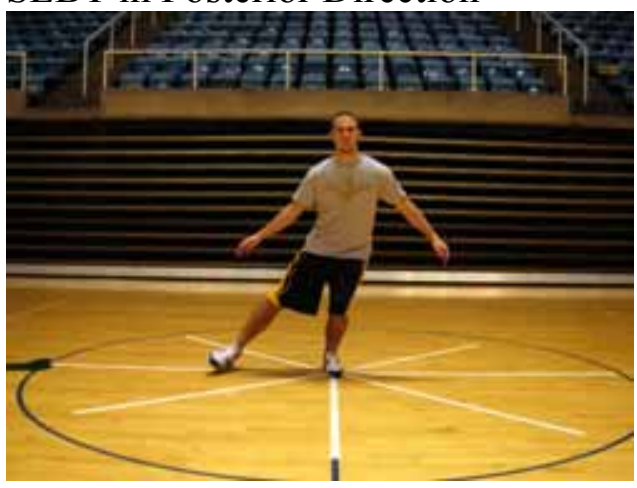

SEBT in Medial Direction
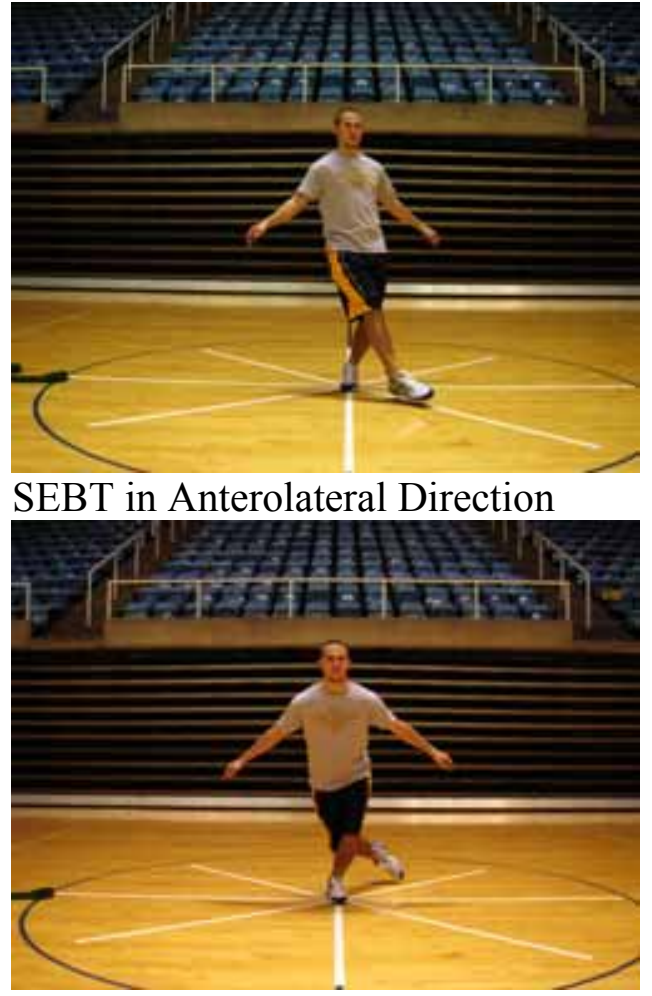

SEBT in Posterolateral Direction

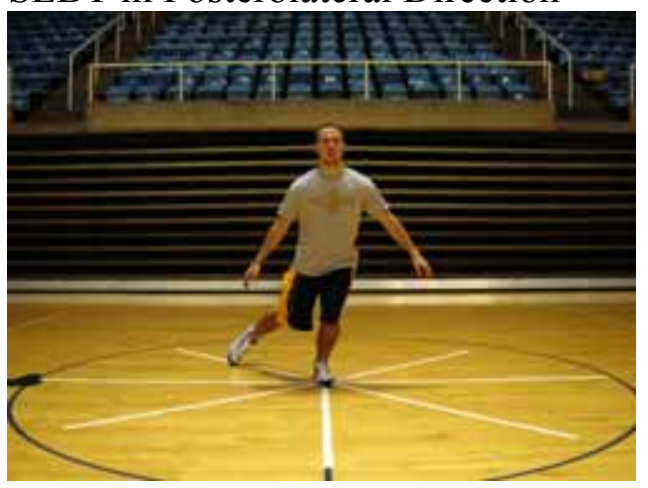

SEBT in Posteromedial Direction

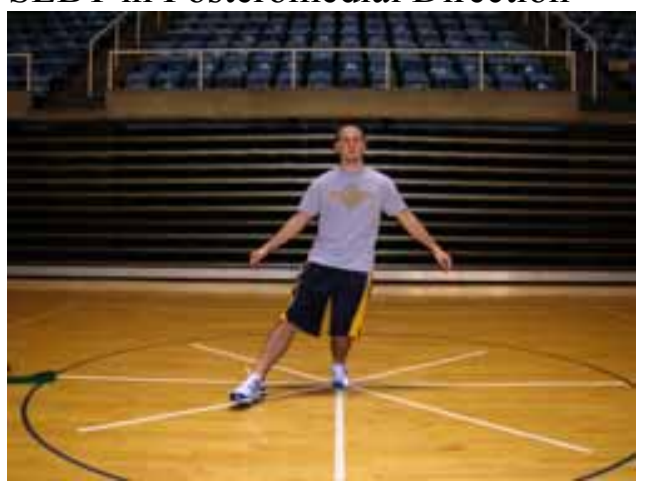

SEBT in Anteromedial Direction 
Figure C4. Diagram of Fatiguing Protocol ${ }^{14,15}$

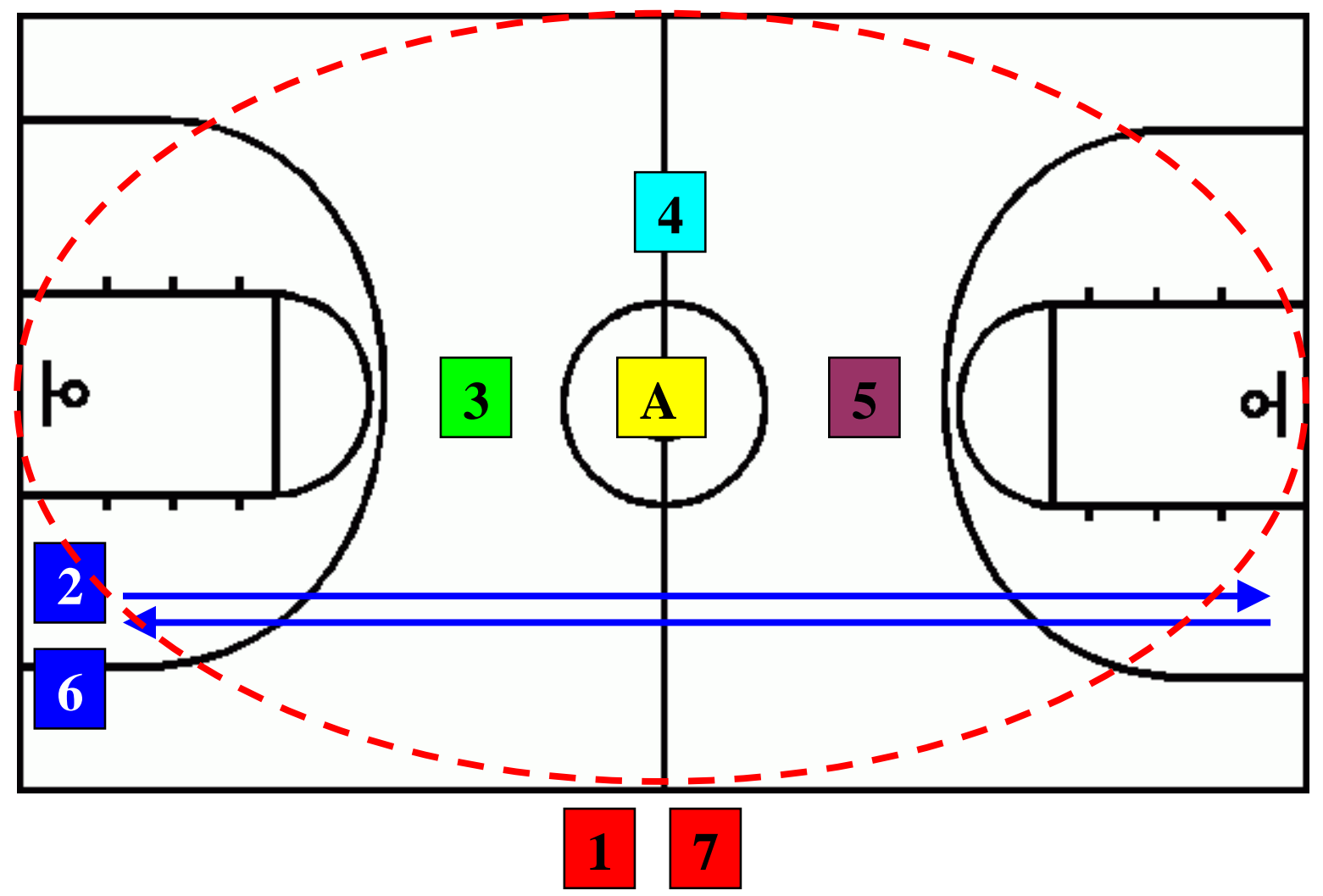

Station 1: five-minute moderate jog at the subjects self-selected pace; Station 2: three minutes of sprints up and down the length of a basketball court; Station 3: two minutes of push-ups; Station 4: two minutes of sit-ups; Station 5: three minutes of 12 inch step-ups; Station 6: three minutes of sprints up and down the basketball court; Station 7: two minute run at as fast a pace as the participant can maintain for the entire two minutes; Station "A": Where the Star Excursion Balance Test will be performed and where the principal investigator will monitor and time the test. 


\section{APPENDIX D}

\section{ADDITIONAL RESULTS}

Table D1. Descriptive Statistics for Star Excursion Balance Test (cm)

\begin{tabular}{lll}
\hline & Pre-Test & Post-Test \\
\cline { 2 - 3 } Anterior & $97.073 \pm 3.632$ & $75.009 \pm 5.198$ \\
Anterolateral & $96.581 \pm 4.683$ & $75.778 \pm 5.287$ \\
Lateral & $95.902 \pm 5.100$ & $75.415 \pm 5.383$ \\
Posterolateral & $97.695 \pm 3.447$ & $79.237 \pm 4.486$ \\
Posterior & $99.748 \pm 4.064$ & $81.041 \pm 4.785$ \\
Posteromedial & $101.021 \pm 4.617$ & $82.574 \pm 5.059$ \\
Medial & $99.408 \pm 4.186$ & $80.169 \pm 4.658$ \\
Anteromedial & $97.733 \pm 3.864$ & $75.831 \pm 4.840$ \\
\hline
\end{tabular}

Table D2. Within subjects ANOVA for the Star Excursion Balance Test

\begin{tabular}{lllll}
\hline & F-Value & $\mathrm{P}$ & ES & $\beta$ \\
\cline { 2 - 5 } Time & 555.675 & $<.001^{*}$ & .934 & 1.000 \\
Direction & 49.010 & $<.001^{*}$ & .557 & 1.000 \\
Time x Direction & 9.487 & $.004^{*}$ & .196 & .862 \\
\hline
\end{tabular}

* significant at the .05 level

Legend: $\mathrm{P}=$ significance, $\mathrm{ES}$ - effect size, $\beta=$ power

Table D3. Pairwise Comparisons for the Star Excursion Balance Test

Pre-Anterior, Post-Anterior

Pre-Anterolateral, Post Anterolateral

Pre-Lateral, Post-Lateral

Pre-Posterolateral, Post-Posterolateral

Pre-Posterior, Post-Posterior

Pre-Posteromedial, Post-Posteromedial

Pre-Medial, Post-Medial

Pre-Anteromedial, Post-Anteromedial

*significance at the .05 level

Legend: $\mathrm{t}=\mathrm{t}$-test, $\mathrm{P}=$ significance

\begin{tabular}{cl}
$\mathrm{t}$ & $\mathrm{P}$ \\
\hline 22.381 & $<.001^{*}$ \\
20.258 & $<.001^{*}$ \\
20.239 & $<.001^{*}$ \\
20.738 & $<.001^{*}$ \\
20.558 & $<.001^{*}$ \\
18.137 & $<.001^{*}$ \\
21.914 & $<.001^{*}$ \\
22.282 & $<.001^{*}$
\end{tabular}
$<.001^{*}$ 
Table D4. Descriptive Statistics for Rating of Perceived Exertion

\begin{tabular}{ll}
\hline & RPE Value \\
\cline { 2 - 2 } Pre-Test & $6.30 \pm .464$ \\
Mid-Test & $12.55 \pm 1.085$ \\
Post-Test & $17.33 \pm .764$ \\
\hline
\end{tabular}

Table D5. Within subjects ANOVA for Rating of Perceived Exertion

\begin{tabular}{lllll}
\hline & F-Value & P & ES & $\beta$ \\
RPE & 2436.147 & $<.001^{*}$ & .984 & 1.000 \\
\hline
\end{tabular}

* significant at the .05 level

Legend: $\mathrm{P}=$ significance, $\mathrm{ES}-$ effect size, $\beta=$ power

$\underline{\text { Table D6. Pairwise Comparisons for the Rating of Perceived Exertion }}$

\begin{tabular}{lll}
\hline & $\mathrm{t}$ & $\mathrm{P}$ \\
\cline { 2 - 2 } Pre- Test to Mid-Test & -33.749 & $<.001^{*}$ \\
Pre-Test to Post-Test & -73.631 & $<.001^{*}$ \\
Mid-Test to Post-Test & -35.037 & $<.001^{*}$ \\
\hline
\end{tabular}

*significance at the .05 level

Legend: $\mathrm{t}=\mathrm{t}$-test, $\mathrm{P}=$ significance 


\section{APPENDIX E}

\section{RECOMMENDATIONS FOR FUTURE RESEARCH}

1. More research in general needs to be conducted looking at activity related or functional fatigue and how it affects dynamic postural control. We understand how dynamic postural control is affected by an isometric fatiguing protocol; however this data is not easily generalizable to the athletic population.

2. In the future use something besides the rating of perceived exertion to guarantee fatigue in the participants. A dynamometer could be used to measure local fatigue

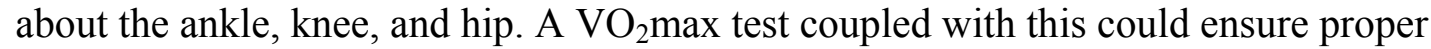
cardiovascular endurance as well. In addition, taking blood samples to detect blood lactate and other fatigue metabolites.

3. Actually using athletes in this study may lead to more clinically related findings. Athletes were not available for use in this study, but one could hypothesize that athletes may already posses the ability to work under a fatigued condition.

4. Using an actual practice or competition instead of a fatiguing protocol may help demonstrate exactly what fatigue an athlete may experience during competition in their sport. However, this type of protocol would be difficult to standardize.

5. Perhaps testing dynamic postural control using the Star Excursion Balance Test while on a force plate may help gain further knowledge into forces on the stance leg and the anterior/posterior and medial/lateral sway movement of the stance foot while reaching in a fatigued state.

6. Once a grasp on the mechanism has been made, develop a training study that looks at potential ways to help train athletes to deal with fatigue in a more efficient way. These studies could look at greater cardiovascular endurance training, advanced core stability programs, increased weight lifting for both power and endurance, and a combination of the three. This data could prove useful for the strength and conditioning coaches and athletic trainers when they are developing pre-season workout programs for their athletes. 


\section{ADDITIONAL REFERRENCES}

39. Lephart S, Picivero D, Giralso J, Fu F. The role of proprioception in the management and rehabilitation of athletic injuries. Am J Sports Med. 1997;25(1):130-137.

40. Drouin JM, Houglum PA, Perrin DH, Gansneder BM. Weight-bearing and non-weightbearing knee-joint reposition sense and functional performance. J Sport Rehabil. 2003;12:54-66.

41. Miller PK, Bird AM. Localized muscle fatigue and dynamic balance. Percept Mot Skills. 1976;42:135-8.

42. Vuillerme N, Nougier V, J Prier J. Can vision compensate for a lower limbs muscular fatigue for controlling posture in humans. Neuroscience Letters. 2001;308:103-106.

43. Sherwood L. Human physiology: from cells to systems; fourth edition. Brooks/Cole: United States. 2001:G1-G15.

44. Riemann BL, Caggiano NA, Lephart SM. Examination of a clinical method of assessing postural control during a functional performance task. J Sport Rehabil. 1999;8:171-183.

45. Riemann BL, Lephart SM. The sensorimotor system, part I: the physiologic basis of functional joint stability. J Athl Train. 2002;37(1):71-79.

46. Johnston H, Howard M, Cawley P, Losse G. Effect of lower extremity muscular fatigue on motor control performance. Med Sci Sports Exerc. 1998;30(12):1702-1707.

47. Prentice WE, Voight MI. Techniques in musculoskeletal rehabilitation. McGraw-Hill Publishing: New York. 2001:49-128.

48. K. Kotsko, oral communication, April 2005

49. Demeritt KM, Shultz SJ, Docherty CL, Gansneder BM, Perrin DH. Chronic ankle instability does not affect lower extremity functional performance. $J$ Athl Train. 2002;37(4):507-511.

50. Rowe A, Wright S, Nyland J, Caborn DNM, Kling R. Effects of a 2-hour cheerleading practice on dynamic postural stability, knee laxity, and hamstring extendibility. $J$ OrthopPhys Ther. 1999;29(8):455-462. 
51. Myers JB, Guskiewicz KM, Schneider RA, Prentice WE. Proprioception and neuromuscular control of the shoulder after muscle fatigue. J Athl Train. 1999;34(4):362-367.

52. Sargant D. Proprioception: how does it work? Australasian J Podiatric Medicine. 2000;34(3):86-92.

53. Grigg P. Peripheral neural mechanisms in proprioception. J Sport Rehabil. 1994;3:217.

54. Ives JC. Neuromuscular control 663-33900. Fall 2003. Ithaca College, Ithaca NY.

55. Irrgang J, Whitney S, Cox E. Balance and proprioceptive training for rehabilitation of the lower extremity. J Sport Rehabil. 1994;3:68-83.

56. Gardner E, Haddad B. pathways to the cerebral cortex for afferent fibers from the hindleg of the cat. Am J Phyisiol. 1953;172:475-482.

57. Clark FJ, Landgren S, Silfvenius H. Projections to the cat's cerebral cortex from low threshold joint afferents. Acta Physiol Scand. 1973;89:504-521.

58. Dye SF. The functional anatomy of the cerebellum: an overview. In: Lephart SM, Fu FH, eds. Proprioception and Neuromuscular Control in Joint Stability. Human Kinetics:Champaign, IL:31-35

59. Jankowska E. Interneuronal relay in spinal pathways from proprioceptors. Prog Neurobiol. 1992;38:335-378.

60. Proske U. What is the role of muscle receptors in proprioception? Muscle Nerve. 2005;31:780-787.

61. Olmsted LC, Carcia CR, Hertel J, Shultz SJ. Efficacy of the star excursion balance tests in detecting reach deficits in subjects with chronic ankle instability. J Athl Train. 2002;37(4):501-506.

62. Ding J, Wexler A, Binder-Macleod S. A predictive model of fatigue in human skeletal muscles. J Appl Physiol. 2002;98:1322-1332.

63. Mcardle WD, Katch FI, Katch VL. Exercise physiology; fifth edition. Lippincott Williams and Wilkins: New York: 400-402.

64. Dalakas MC, Mock V, Hawkins MJ. Fatigue: definitions, mechanisms, and paradigms for study. Seminars in Oncology. 1998;25(1)suppl:48-53. 
65. Johnston H, Howard M, Cawley P, Losse G. Effect of lower extremity muscular fatigue on motor control performance. Med Sci Sports Exerc. 1998;30(12):1702-1707.

66. Sparto PJ, Parnianpour M, Reinsel TE, Simon S. The effect of fatigue on multijoint kinematics, coordination, and postural stability during a repetitive lifting task. $J$ OrthopPhys Ther. 1997;25(1):3-11. 